\title{
Origin of band and localized electron states in photoemission of $\mathrm{NiO}$
}

\author{
Jan Bała \\ Institute of Physics, Jagellonian University, Reymonta 4, PL-30059 Kraków, Poland \\ Andrzej M. Oleś \\ Institute of Physics, Jagellonian University, Reymonta 4, PL-30059 Kraków, Poland \\ and Max-Planck-Institut für Festkörperforschung, Heisenbergstrasse 1, D-70569 Stuttgart, Federal Republic of Germany \\ Jan Zaanen \\ Institute Lorentz for Theoretical Physics, Leiden University, P.O.B. 9506, NL-2300 RA Leiden, The Netherlands
}

(Received 1 June 1999; revised manuscript received 3 January 2000)

\begin{abstract}
In a variety of model studies it has been shown that the problem of a single hole in a Mott insulator can be quite well addressed by assuming that all that matters is the interaction between the propagating hole and the spin waves of the insulator. $\mathrm{NiO}$ has been often taken as the archetypical example of a Mott insulator and recent angular resolved photoemission studies have revealed that holes in this material share both itinerant and localized aspects that are very hard to understand either in conventional band-structure theory or from purely localized approaches. Starting from a strongly coupled electronic multiband Hubbard model, we derive a generalized strong-coupling spin-fermion model. The model includes the multiplet structure of the electronic excitations and describes the interaction of the $\mathrm{O}(2 p)$ holes moving in oxygen bands with the spins localized on $\mathrm{Ni}$ ions. In linear spin-wave order we find an effective Hamiltonian describing the scattering of the bandlike holes on the spin waves. This problem is solved in rainbow order, and we find that the outcomes resemble well the experimental findings. In contrast to earlier impurity interpretations stressing spatial locality, we find that momentum dependencies are dominating the hole dynamics.
\end{abstract}

\section{SPIN WAVES AND PHOTOEMISSION OF NiO}

The discovery of the high- $T_{c}$ superconductors triggered a revival in the interest of the electronic structure of the transition-metal oxides. Several interesting electronic and magnetic properties observed in these materials are caused by strong electron correlations, driven by the large Coulomb interaction $U$ between the $3 d$ electrons. Not surprisingly, band-structure theory fails in even the most elementary aspects. For instance, the calculations performed within local (spin) density approximation [L(S)DA] predict that some of the transition-metal oxides (such as $\mathrm{FeO}$ and $\mathrm{CoO}$ ) are metals, while in reality they are large-gap Mott insulators. Even if the insulating character, as for $\mathrm{NiO}$, is correctly reproduced, the value of the gap is too small by an order of magnitude. ${ }^{1}$

Some time ago, it seemed that this problem was basically solved in approaches that emphasized the interactions. It was assumed that the physics was essentially local; single electron momentum was supposed to be destroyed completely and instead one could limit the description to (atomic) interactions and short-range quantum delocalization. In this way the momentum integrated spectral functions could be explained in some detail. ${ }^{2,3}$ Most importantly, the so-called satellites seen in the photoemission spectra (spectral weight showing up at large excitation energies), originally discussed in terms of spectroscopic artifacts, were identified to correspond with Hubbard bands, showing that Hubbard models can be taken quite literally, even on energy scales for which they were not intended.
Because this "Hubbard band structure" was now accessible experimentally, ${ }^{4,5}$ surprises followed. It turned out that other bands could show up in between the $3 d$ derived Hubbard bands-in the late $3 d$ compounds this is generically the ligand $2 p$ band, with the effect that the top of the valence band is of $p$ character, resulting in a charge-transfer (CT) insulator. Especially in $\mathrm{NiO}$ one has at first sight a problem with such an interpretation since the top of the valence band seems to reflect the multiplet splittings of the $3 d^{7}$ lower Hubbard band (LHB). This problem is resolved within this "localized" point of view if the effects of covalency are taken into account. Even if the finite bandwidth of the $2 p$ states is taken seriously ("impurity approximation"), the hybridization between the LHB and the $p$ band is strong enough to pull out bound states above the top of the latter. These bound states can exist in the different spin- and angular-momentum channels of the $3 d$ "impurity,", thereby creating a bound-state structure resembling that of the bare LHB, which itself shows up as a high-energy satellite.

This way of thinking was formally justified by Zhang and Rice. 6 They showed that the "gap-state"' binding energy could be much larger than the bandwidth related to the delocalization of these bound states; it is correct to assume that the carriers in these materials carry the local quantum numbers of the LHB [e.g., a hole in "Ni(II)" is either a low- or high-spin "Ni(III)" state]. Only if one invests enough energy to exceed the Zhang-Rice (ZR) binding energy, it becomes consequential that these carriers originate predominantly from ligand states.

The angular-resolved photoemission (ARPES) study on $\mathrm{NiO}$ by Shen et al. ${ }^{5}$ constituted an important next step. Despite the successes of the localized approach we just discussed, these data revealed that the electronic structure of 
$\mathrm{NiO}$ is surprisingly bandlike. Especially at higher energies, rather well-defined dispersing states were observed, which seemed to be naturally explained from conventional bandstructure theory ${ }^{5}$ rather than from a local, strongly correlated point of view. It is noticed that $\mathrm{NiO}$ is special in this regard, because, e.g., cuprate ARPES spectra do not reveal dispersive features at higher energies.

Here we will present a theory of a different kind, which seems to reconcile in a natural way the dichotomy of the "band" and "localized" nature of the valence-band electronic structure of $\mathrm{NiO}$. We start from the fact that $\mathrm{NiO}$ is a CT insulator of the large- $U$ variety. We even overemphasize the interactions by adopting the above spin-fermion limit that is not quite realized in this case. The ground state of $3 d$ spin system is a classical antiferromagnet and therefore its elementary excitation spectrum is understood in great detail in terms of linear spin-wave (LSW) theory. Accordingly, the problem in $\mathrm{NiO}$ is that of the oxygen hole scattering off these spin waves via the mediating Kondo interactions. Mathematically, this interaction is similar to standard electronphonon (polaron) theory. However, the couplings are very strong and the interaction vertex shows extreme momentum dependence, contrasting sharply to the locality characterizing phonon problems.

We lean heavily on the work done in the context of the $t-J$ model. This latter model describes the hopping of a (featureless) hole in a Heisenberg spin system. The $t-J$ model seems at first sight simpler than the spin-fermion model, but it actually describes a more extreme situation. The hole can only delocalize by exciting spins and a bare kinetic-energy scale is absent: the coupling constant is in fact diverging. Although the physics at any finite hole density is still unclear, it is now generally believed that the one-hole case is understood in detail, precisely in terms of the above spinwave framework. The late Schmitt-Rink, together with Ruckenstein and Varma, were the first to realize ${ }^{7}$ that the one-hole problem could be treated by rather conventional means, because of the classical nature of the spin vacuum. The Néel state is qualitatively not different from any crystalline solid except that spins (especially $S=1 / 2$, but also the $S=1$ spins of $\mathrm{NiO}$ ) are much more quantum mechanical than even the lightest ions. However, one is still above the lower critical dimensionality of the classical mean-field theory and the Gaussian fluctuations (spin waves) can account well for the quantum corrections. One fermion cannot change the nature of the vacuum and, at least asymptotically, the spin waves are also, in this case, all that matters. Evaluating the $t-J$ model up to LSW order (see also Sec. II) yields a Hamiltonian similar to the one used in the context of $\mathrm{CuO}_{2}$ (Ref. 8) and looks like that of an electron coupling to acoustic phonons except that the bare kinetic energy of the former is missing.

The topology of the quasiparticle $(\mathrm{QP})$ band in the $t-J$ model is that of a particle hopping on a magnetic sublattice, while the QP bandwidth $\sim z J$, is set by the scale of the spin fluctuations. ${ }^{9}$ The total extent of the spectrum turns out to be $\sim z t$ (i.e., the hole-magnon interaction scale) while the QP pole strength is $a_{\mathbf{k}} \sim J / t$, as expected. Physically this QP is different from a normal polaron; the hopping of the hole $(\sim t)$ acts to increase locally the quantum spin fluctuations, melting the "spin solid" in its immediate neighborhood. The motion of the hole is accompanied by a backflow of spin fluid, ${ }^{10}$ allowing it to move coherently throughout the lattice ("spin-liquid polaron").

Because of the strong coupling, one needs a selfconsistent theory and under the neglect of vertex corrections this becomes the rainbow resummation [or self-consistent Born approximation $^{7}$ (SCBA)], where one uses the fully dressed hole Green's function to calculate the hole selfenergy (Sec. II). Assuming that the low-energy part of the hole spectrum is dominated by a QP pole, it was shown by Kane, Lee, and Read ${ }^{11}$ that the self-consistently calculated Green's function has indeed a QP peak accompanied by an incoherent part.

In principle, the above theory is devised to describe the long-wavelength limit. Important for our purposes, detailed comparisons of the outcomes of this theory with exact diagonalization studies of small clusters, ${ }^{12}$ which were next extended to the spectral functions of real particles added to (removed from) the antiferromagnetic (AF) state,${ }^{13}$ show that a nearly perfect description is obtained for the whole spectral function, including the incoherent, multiple spin-wave contributions at energies $\omega>z J$. This can be understood to some extent by considering the Ising $t-J_{z}$ limit, which is the worst case because even the emergent kinetic scale disappears. LSW-SCBA reproduces essentially correctly ${ }^{14}$ the characteristic ladder spectrum of the nonretraceable path approximation, ${ }^{15}$ and it is well known that the corrections ("Trugman loops" ${ }^{16}$ ) are not important for the overall shape of the spectrum.

Coming back to the situation in $\mathrm{NiO}$ as described with a spin-fermion model, LSW-SCBA should in principle be a better approximation than for the $t-J$ model. First, the $S=1$ antiferromagnet is closer to the classical (large $S$ ) limit than its $S=1 / 2$ counterpart. More importantly, the coupling constant is no longer infinite because of the finite bare bandwidth of the $2 p$ holes. Nevertheless, the outcomes have to be more complex. In LSW language, bound states occur due to a local binding of the oxygen hole to a magnon. This composite particle should hop as a whole through the AF lattice, thereby producing the QP pole, as well as the multiple spinwave incoherent background. Finally, at even higher energies it is expected that $\mathbf{k}$-dependent features exist reflecting the bare oxygen bands. Rather remarkably, this is precisely what LSW-SCBA yields, as shown by our preliminary results reported earlier. ${ }^{17,18}$

The paper is organized as follows. In Sec. II we consider a two-dimensional (2D) slab to represent the (001) surface of $\mathrm{NiO}$ and we derive a generalized spin-fermion model that we believe is sufficiently realistic to be used to interpret the whole valence-band region. This incorporates the full magnetic structure, as well as all orbital degrees of freedom of relevance in planar directions for the valence band. We include both oxygen " $\sigma$ " and " $\pi$ " orbitals, and the degeneracy of the $3 d$ shell ( $e_{g}$ and $t_{2 g}$ orbitals), with the Hund's rule interactions in the intermediate states. Under the condition that $d-d$ excitons do not play a dynamical role, we show that this reduces to a spin-fermion model, albeit a quite complicated one involving more $p$ bands, AF as well as ferromagnetic (FM) hole-spin interactions, etc. We motivate our choices for the free parameters in the model by spectroscopic data. Evaluating this model numerically up to LSW-SCBA 
order (Sec. III) we find a description of NiO's single-hole spectral function that accounts semiquantitatively for the angular-resolved photoemission data of this material. The paper is summarized in Sec. IV. The Appendix contains the symmetry arguments that allows us to interpret the selfenergy obtained at different momenta.

\section{SPIN-FERMION MODEL FOR NiO}

\section{A. Effective Hamiltonian}

$\mathrm{NiO}$ is best characterized as a CT insulator, ${ }^{19}$ where the $\mathrm{O}(2 p)$ band lies in between the $\mathrm{Ni}(3 d)$ upper Hubbard band (UHB) and LHB. If the splittings between these bands is large enough compared to the $d$-p hybridization $\left(t_{p d}\right)$, one finds that the Ni holes localize, and an oxygen (doped) hole interacts with the $S=1 \quad\left({ }^{3} A_{2}\right)$ spins on the neighboring $\mathrm{Ni}$ ions. We assume a small hybridization compared with the Coulomb interaction $U$ and the CT energy $\Delta=\varepsilon_{d}-\varepsilon_{p}$, where $\varepsilon_{d}$ and $\varepsilon_{p}$ are the energies of $3 d$ and $2 p$ states, respectively. In the regime of $t_{p d} \ll \Delta$ and $t_{p d} \ll U-\Delta$, the effective interactions between an oxygen hole and $\mathrm{Ni} S=1$ spins can be derived using perturbation theory. ${ }^{20} \mathrm{~A}$ moving hole may scatter against spin or orbital excitations. The latter have excitation energies of at least $1 \mathrm{eV}$ and may be safely neglected.

It is well known that the electronic states described by the three-band model $^{21}$ for high-temperature superconducting oxides correspond to those treated in an effective $t-J$ model in the strongly correlated limit. ${ }^{6}$ One possibility is therefore to extend the $t-J$ model by the hopping to further neighbors that follows from a mapping procedure to the effective space of a single band. ${ }^{22}$ While the $t-J$ model is a generic Hamiltonian, another and more realistic route may be followed by considering spin-fermion models that result from the full electronic structure in the strongly correlated regime, if both the symmetry of the doped holes and the multiplet structure of $3 d$ states are taken into account. ${ }^{10,23}$ If the Coulomb interactions are strong enough, it is in general possible to integrate out perturbatively the $3 d$ charge degrees of freedom. The problem that remains is that of a carrier that is strongly coupled to the spin background.

Undoped $\mathrm{NiO}$ is ionic, and one has $\mathrm{Ni}^{2+}$ ions in the highspin ${ }^{3} A_{2}$ configuration, while the $2 p$ states of oxygen ions are filled. It is convenient to represent the components of the local $d^{8}$ triplets $\left({ }^{3} A_{2}\right)$ by hard-core bosons, ${ }^{24}$

$$
\begin{gathered}
B_{i 1}^{\dagger}=d_{x \uparrow}^{\dagger} d_{z \uparrow}^{\dagger}, \\
B_{i 0}^{\dagger}=\frac{1}{\sqrt{2}}\left(d_{x \uparrow}^{\dagger} d_{z \downarrow}^{\dagger}+d_{x \downarrow}^{\dagger} d_{z \uparrow}^{\dagger}\right), \\
B_{i,-1}^{\dagger}=d_{x \downarrow}^{\dagger} d_{z \downarrow}^{\dagger} .
\end{gathered}
$$

There are two classes of excited states by which the spin of an oxygen hole may be exchanged with one of the spins in the background. Either one of the holes in the $d^{8}$ triplet jumps out to a neighboring oxygen site and a $d^{9}$ configuration is created, or a doped hole moves to the transition-metal site that results in the $d^{7}$ intermediate state. In the UHB of $d^{9}$ configurations one has singly occupied $e_{g}$ orbitals. These excited states are characterized by the energy $\Delta$, if the small corrections due to the energy difference between the $d_{x^{2}-y^{2}}$ and $d_{3 z^{2}-r^{2}}$ levels are neglected.

The LHB that consists of $d^{7}$ configurations is more complex. First of all, an oxygen hole moving to a Ni site may create a high-spin $(S=3 / 2){ }^{4} T_{1 g}$ state when it occupies one of the $t_{2 g}\left(d_{i t \sigma}^{\dagger}\right)$ orbitals,

$$
\begin{gathered}
{ }^{4} T_{i, 3 / 2} \sim d_{i t \uparrow}^{\dagger} B_{i 1}^{\dagger}, \\
{ }^{4} T_{i, 1 / 2} \sim \frac{1}{\sqrt{3}}\left(d_{i t \downarrow}^{\dagger} B_{i 1}^{\dagger}+\sqrt{2} d_{i t \uparrow}^{\dagger} B_{i 0}^{\dagger}\right), \\
{ }^{4} T_{i,-1 / 2} \sim \frac{1}{\sqrt{3}}\left(d_{i t \uparrow}^{\dagger} B_{i,-1}^{\dagger}+\sqrt{2} d_{i t \downarrow}^{\dagger} B_{i 0}^{\dagger}\right), \\
{ }^{4} T_{i,-3 / 2} \sim d_{i t \downarrow}^{\dagger} B_{i,-1}^{\dagger} \cdot
\end{gathered}
$$

In addition to this high-spin state, one has as well two lowspin doublet states with either a $t_{2 g}\left({ }^{2} T_{1 g}\right)$ or an $e_{g}\left({ }^{2} E_{g}\right)$ orbital occupied by an extra hole,

$$
\begin{gathered}
{ }^{2} T_{i, 1 / 2} \sim \frac{1}{\sqrt{3}}\left(\sqrt{2} d_{i t \downarrow}^{\dagger} B_{i 1}^{\dagger}-d_{i t \uparrow}^{\dagger} B_{i 0}^{\dagger}\right), \\
{ }^{2} T_{i,-1 / 2} \sim \frac{-1}{\sqrt{3}}\left(\sqrt{2} d_{i t \uparrow}^{\dagger} B_{i,-1}^{\dagger}-d_{i t \downarrow}^{\dagger} B_{i 0}^{\dagger}\right),
\end{gathered}
$$

and two ${ }^{2} E$ configurations,

$$
\begin{gathered}
{ }^{2} E_{i, 1 / 2} \sim B_{i 1}^{\dagger} d_{i x(z) \downarrow}^{\dagger}, \\
{ }^{2} E_{i,-1 / 2} \sim d_{i x(z) \uparrow}^{\dagger} B_{i-1}^{\dagger} .
\end{gathered}
$$

As a result of the transitions to all these excited states one finds the Kondo-like exchange interactions, originating from the virtual hopping of the $p$ hole into the Hubbard bands. ${ }^{20}$ Furthermore, in order to make a comparison with a $\mathrm{NiO}(001)$ surface measured in the ARPES experiments by Shen et al., we restrict ourselves to a single 2D slab, mimicking the (001) surface of $\mathrm{NiO}$. By considering the virtual transitions to higher-energy excitations, one finds that the electronic states of the $\mathrm{NiO}(001)$ plane are described by the following spin-hole model:

$$
H=H_{s}+H_{h}^{0}+H_{h}^{J}+H_{h}^{A F}+H_{h-s} .
$$

Without holes the system is described by the superexchange Hamiltonian for $S=1$ spins on $\mathrm{Ni}$ ions,

$$
H_{s}=J \sum_{\langle i j\rangle \in(A, B),(C, D)} \mathbf{S}_{i} \cdot \mathbf{S}_{j}-J^{\prime} \sum_{\langle i j\rangle \in(A, C),(B, D)} \mathbf{S}_{i} \cdot \mathbf{S}_{j},
$$

where an AF interaction $J$ couples the pairs of next-nearest neighbor spins $\mathbf{S}_{i}$ along the $x$ and $y$ directions, being separated by one oxygen ion [see Fig. 1(b)]. These interactions stabilize an AF ground state with two pairs of interpenetrating magnetic sublattices, $(A, B)$ and $(C, D)$, occupied by up ( $A$ and $C$ ) and down ( $B$ and $D)$ spins in the respective Néel state. The weak FM interaction, $J^{\prime}=\alpha J$, couples the nearest neighbors, i.e., acts between pairs of spins that belong to $(A, C)$ and $(B, D)$ sublattices, respectively, and is frustrated 
(a)

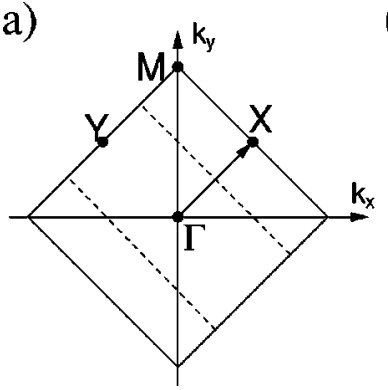

(b)

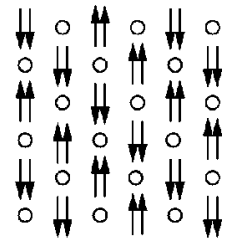

FIG. 1. The BZ (a) for the NiO (001) plane, shown schematically in part (b), with two holes at each $\mathrm{Ni}$ ion indicated by arrows. The dashed lines in (a) indicate the folding of the $\mathrm{BZ}$ in the AF phase, where $\Gamma-X$ shows the nesting vector.

in the AF state. The spin Hamiltonian with $S=1$ may be expressed in terms of two Schwinger bosons $b_{i \sigma}$ at each site (Ref. 11), and then is easily diagonalized by considering the fluctuations around the classical saddle point,

$$
\begin{gathered}
b_{i \downarrow}=b_{j \uparrow}=\sqrt{2 S}, \\
b_{i \uparrow}=b_{j \downarrow}=0,
\end{gathered}
$$

where $i \in A, C$ and $j \in B, D$. This gives one branch of acoustic magnons with energies $\omega_{\mathbf{q}}$ in unfolded Brillouin zone (BZ),

$$
H_{s}=\sum_{\mathbf{q}} \omega_{\mathbf{q}} \beta_{\mathbf{q}}^{\dagger} \beta_{\mathbf{q}},
$$

with magnon energies $\omega_{\mathbf{q}}$ given by

$$
\omega_{\mathbf{q}}=4 J\left[\left(1-\alpha \zeta_{\mathbf{q},+}\right)^{2}-\left(\gamma_{\mathbf{q}}-\alpha \zeta_{\mathbf{q},-}\right)^{2}\right]^{1 / 2},
$$

and with $\gamma_{\mathbf{q}}=\frac{1}{2}\left(\cos 2 q_{x}+\cos 2 q_{y}\right), \zeta_{\mathbf{q}, \pm}=\frac{1}{2} \cos \left(q_{x} \pm q_{y}\right)$ (defining the lattice distance between $\mathrm{Ni}$ and $\mathrm{O}$ nearest neighbors as a unit length $a=1$ ). The spin waves with their creation operators $\beta_{\mathbf{q}}^{\dagger}$ are described by the Bogoliubov transformation,

$$
\begin{gathered}
\beta_{\mathbf{q}}=u_{\mathbf{q}} b_{\mathbf{q}}-v_{\mathbf{q}} b_{-\mathbf{q}}^{\dagger}, \\
u_{\mathbf{q}}=\left[\frac{1+\left(1-x_{\mathbf{q}}^{2}\right)^{1 / 2}}{2\left(1-x_{\mathbf{q}}^{2}\right)^{1 / 2}}\right]^{1 / 2}, \\
v_{\mathbf{q}}=-\operatorname{sgn}\left(B_{\mathbf{q}}\right)\left[\frac{1-\left(1-x_{\mathbf{q}}^{2}\right)^{1 / 2}}{2\left(1-x_{\mathbf{q}}^{2}\right)^{1 / 2}}\right]^{1 / 2},
\end{gathered}
$$

where $x_{\mathbf{q}}=B_{\mathbf{q}} / A_{\mathbf{q}}, A_{\mathbf{q}}=J-J^{\prime} \zeta_{\mathbf{q},+}, B_{\mathbf{q}}=J \gamma_{\mathbf{q}}-J^{\prime} \zeta_{\mathbf{q},-}$, and $b_{\mathbf{q}}$ is a Fourier transform of the Schwinger boson operators in real space (7),

$$
b_{\mathbf{q}}=\sum_{i \in A, C} b_{i \uparrow} e^{i \mathbf{q} \cdot \mathbf{r}_{i}}+\sum_{j \in B, D} b_{j \downarrow} e^{i \mathbf{q} \cdot \mathbf{r}_{j}}
$$

Doped holes that occupy the $\mathrm{O}(2 p)$ orbitals represent the second subsystem. We label the $\mathrm{O}(2 p)$ orbitals that are oriented along $x$ or $y$ axes in the (001) plane as $x(y)$ and the direct oxygen-oxygen hoppings are described by $H_{h}^{0}$,

$$
\begin{aligned}
H_{h}^{0}= & \sum_{m n \sigma}\left[t_{x x}\left(a_{m, x \sigma}^{\dagger} a_{n, x \sigma}+a_{m, y \sigma}^{\dagger} a_{n, y \sigma}\right)\right. \\
& \left.+t_{x y}\left(a_{m, x \sigma}^{\dagger} a_{n, y \sigma}+a_{m, y \sigma}^{\dagger} a_{n, x \sigma}\right)\right],
\end{aligned}
$$

where $a_{m, x(y) \sigma}^{\dagger}$ are creation operators for a hole at site $m$ with spin $\sigma=\uparrow$ or $\downarrow$ on orbitals along $x(y)$ direction.

We claim that, as in a high-temperature superconductor, a hole doped to an oxygen orbital in $\mathrm{NiO}$ interacts with the spins on the neighboring transition metal ions by Kondo-like exchange interactions. They are derived by considering the intermediate $d^{9}$ and $d^{7}$ states at $\mathrm{Ni}$ ions and the corresponding hole configuration (one or three holes) on a $\mathrm{Ni}$ ion, which occur, respectively, when the hole hops from $\mathrm{O}$ to $\mathrm{Ni}$ ion, and vice versa. In $d^{9}$ states one has a hole within one of the $e_{g}$ orbitals. The spectrum of $d^{7}$ configurations is more complex as one finds either a high-spin ${ }^{4} T_{1 g}$ quartet [Eq. (2)], or one of the low-spin doublets, ${ }^{2} T_{1 g}$ [Eq. (3)], and ${ }^{2} E_{g}$ [Eq. (4)], as intermediate states. The $e_{g}$ oxygen orbitals hybridize with the $d_{x^{2}-y^{2}}$ and $d_{3 z^{2}-r^{2}}$ orbitals (both occupied by one hole each in ${ }^{3} A_{1}$ ) by $t_{x}$ and $t_{z}$, respectively, while the $t_{2 g}$ orbitals hybridize only with (unoccupied) $d_{x y}$ orbitals by $t_{\pi}$.

It is convenient to write the three-site hopping and Kondo terms by defining locally $e_{g}$ and $t_{2 g}$ symmetries for the oxygen orbitals by their coupling to the respective $\mathrm{Ni}(3 d)$ states, i.e., pointing either along or perpendicular to Ni-O bonds, respectively. Using this notation, the free oxygen bands $\left(H_{h}^{J}\right)$ are renormalized by a moving hole by the spin-independent part,

$$
H_{h}^{J}=\sum_{i m n \sigma}\left[\left(T_{x, e}+T_{z, e}\right) a_{m, e \sigma}^{\dagger} a_{n, e \sigma}-T_{t} a_{m, t \sigma}^{\dagger} a_{n, t \sigma}\right],
$$

where $m$ and $n$ are nearest neighbors of Ni site $i$,

$$
\begin{gathered}
T_{x(z), e}=\frac{1}{2}\left[J_{K}^{x(z)}(\Delta)-J_{K}^{x(z)}\left({ }^{2} E\right)\right], \\
T_{t}=\frac{1}{3}\left[2 J_{K}\left({ }^{4} T\right)+J_{K}\left({ }^{2} T\right)\right] .
\end{gathered}
$$

The spin-dependent contribution in Eq. (5) is of the form

$$
H_{h}^{A F}=S \sum_{i m n \sigma} \sum_{\xi \xi^{\prime}=e, t} \lambda_{\sigma} J_{\xi \xi^{\prime}}\left[a_{m \xi \sigma}^{\dagger} a_{n \xi^{\prime} \sigma}+\text { H.c. }\right],
$$

with $\lambda_{\sigma}= \pm$ for $\sigma=\uparrow, \downarrow$, respectively. In the reciprocal space $H_{h}^{A F}$ leads to the coupling between the $\mathbf{k}$ and $\mathbf{k}+\mathbf{Q}$ states,

$H_{h}^{A F}=S \sum_{\mathbf{k}} \sum_{\xi \xi^{\prime}=x, y} \alpha_{\xi \xi^{\prime}}(\mathbf{k})\left[a_{\mathbf{k}+\mathbf{Q}, \xi_{\uparrow} \uparrow}^{\dagger} a_{\mathbf{k}, \xi^{\prime} \uparrow}-a_{\mathbf{k}+\mathbf{Q}, \xi_{\downarrow} \downarrow}^{\dagger} a_{\mathbf{k}, \xi^{\prime} \downarrow}\right]$,

where $\mathbf{Q}=(\pi / 2, \pi / 2)$ is the $2 \mathrm{D}$ nesting vector [Fig. 1(a)], and

$$
\begin{gathered}
\alpha_{x x}(\mathbf{k})=-2\left[\left(J_{x, e}+J_{z, e}\right) \sin \left(2 k_{x}\right)-J_{t} \sin \left(2 k_{y}\right)\right], \\
\alpha_{y y}(\mathbf{k})=-2\left[\left(J_{x, e}+J_{z, e}\right) \sin \left(2 k_{y}\right)-J_{t} \sin \left(2 k_{x}\right)\right], \\
\alpha_{x y}(\mathbf{k})=4\left[\left(J_{x, e}-J_{z, e}\right) \cos k_{x} \sin k_{y}+J_{t} \cos k_{y} \sin k_{x}\right], \\
\alpha_{y x}(\mathbf{k})=4\left[\left(J_{x, e}-J_{z, e}\right) \cos k_{y} \sin k_{x}+J_{t} \cos k_{x} \sin k_{y}\right] .
\end{gathered}
$$




$$
\begin{aligned}
& \text { with } J_{x(z), e}=\left[J_{K}^{x(z)}(\Delta)+J_{K}^{x(z)}\left({ }^{2} E\right)\right], \quad J_{t}=\frac{2}{3}\left[J_{K}\left({ }^{4} T\right)\right. \\
& \left.-J_{K}\left({ }^{2} T\right)\right], \text { and } \\
& \qquad \begin{aligned}
J_{K}\left({ }^{4} T\right) & =t_{\pi}^{2} /\left[U\left({ }^{4} T\right)-\Delta+4 D_{q}\right], \\
J_{K}^{x(z)}\left({ }^{2} E\right) & =t_{x(z)}^{2} /\left[U\left({ }^{2} E\right)-\Delta-6 D_{q}\right], \\
J_{K}\left({ }^{2} T\right) & =t_{\pi}^{2} /\left[U\left({ }^{2} T\right)-\Delta+4 D_{q}\right], \\
J_{K}^{x(z)}(\Delta) & =t_{x(z)}^{2} /\left[\Delta+6 D_{q}\right] .
\end{aligned}
\end{aligned}
$$

Compared with the usual spin-fermion model derived from a spin-degenerate Hubbard model, ${ }^{20,25}$ the present model is more complex. Besides the two flavors of fermions $(e, t)$, the multiplet structure of the LHB manifests itself in the form of one FM, $\left[J_{K}\left({ }^{4} T\right)\right]$, and two AF, $\left[J_{K}^{x(z)}\left({ }^{2} E\right)\right]$ and $\left[J_{K}\left({ }^{2} T\right)\right]$, exchange interactions, in addition to the UHB-derived interaction $\left[J_{K}^{x(z)}(\Delta)\right]$. Thus, the ferromagnetic sign of the Kondo interactions is found only for the high-spin $(S=3 / 2)$ states similarly as in the spin-fermion model for the $\mathrm{CuO}_{2}$ plane. ${ }^{8}$

The free-fermion part of the Hamiltonian, $H_{h}^{0}+H_{h}^{J}$, can be easily diagonalized to give two hole bands,

$$
H_{h}=H_{h}^{0}+H_{h}^{J}=\sum_{\mathbf{k}, \mu \sigma} \varepsilon_{\mu}(\mathbf{k}) a_{\mathbf{k}, \mu \sigma}^{\dagger} a_{\mathbf{k}, \mu \sigma}
$$

where

$$
\varepsilon_{e(t)}(\mathbf{k})=2\left[E_{11}+E_{22} \pm \sqrt{\left(E_{11}-E_{22}\right)^{2}+4 E_{12} E_{21}}\right],
$$

with

$$
\begin{gathered}
E_{11}=-t_{x x} \cos k_{x} \cos k_{y}+\left(T_{x, e}+T_{z, e}\right) \sin ^{2} k_{x} \\
-T_{t} \sin ^{2} k_{y}, \\
E_{12}=\left(-t_{x y}-T_{x, e}+T_{z, e}+T_{t}\right) \sin k_{x} \sin k_{y} .
\end{gathered}
$$

The elements $E_{22}$ and $E_{21}$ can be obtained from $E_{11}$ and $E_{12}$ by the transformations $\left\{k_{x} \leftrightarrow k_{y}\right\}$ and $\left\{t_{x x} \leftrightarrow t_{y y}\right\}$. In the $\mathrm{AF}$ background the states with momenta $\mathbf{k}$ and $\mathbf{k}+\mathbf{Q}$ are coupled by $H_{h}^{A F}$, and the full free-hole Hamiltonian $H_{h}^{0}+H_{h}^{J}+H_{h}^{A F}$ leads to the following $4 \times 4$ matrix problem:

$$
\left[\begin{array}{cc}
\left(\left[H_{h}^{0}\right]+\left[H_{h}^{J}\right]\right)(\mathbf{k}) & \lambda_{\sigma}[\alpha](\mathbf{k}) \\
\lambda_{\sigma}[\alpha]^{\dagger}(\mathbf{k}) & \left(\left[H_{h}^{0}\right]+\left[H_{h}^{J}\right]\right)(\mathbf{k}+\mathbf{Q})
\end{array}\right],
$$

where $[\cdots]$ stands for the $2 \times 2$ matrices labeled by spin indices, and the elements of $[\alpha]$ are defined by Eqs. (17).

The coupling between the subsystem of itinerant holes at $\mathrm{O}(2 p)$ orbitals and localized spins at $\mathrm{Ni}$ sites is realized by the Kondo-like spin-hole exchange interactions, originating from the virtual hopping of the $p$ hole into the Hubbard bands, ${ }^{20}$

$$
H_{h-s}=\sum_{i m n}\left[\left(J_{x, e}+J_{z, e}\right) \mathbf{S}_{i} \cdot \mathbf{S}_{m n, e}-J_{t} \mathbf{S}_{i} \cdot \mathbf{S}_{m n, t}\right],
$$

where the (nonlocal) hole spin $(s=1 / 2)$ operators,

$$
\begin{gathered}
s_{m n, s}^{+}=a_{m, s \uparrow}^{\dagger} a_{n, s \downarrow}, \\
s_{m n, s}^{z}=\frac{1}{2}\left(a_{m, s \uparrow}^{\dagger} a_{n, s \uparrow}-a_{m, s \downarrow}^{\dagger} a_{n, s \downarrow}\right),
\end{gathered}
$$

are defined by hole creation operators $a_{m, s \sigma}^{\dagger}$ in a $s=e, t\left(e_{g}\right.$ and $t_{2 g}$ ) orbital at site $m$.

Schmitt-Rink et al. ${ }^{7,11}$ proposed that these kinds of Hamiltonians can be treated using the powerful formalism of polaron theory. The idea is that only the linearized collective excitations (magnons) of the spin system are important. Comparison with exact results for the $t-J$ (Ref. 14) and spin-fermion ${ }^{8,26}$ models shows that this strongly coupled hole-magnon problem is accurately solved already in LSWSCBA, also with regards to the overall structure of the spectral function of a single hole. In LSW order the total Hamiltonian has the following form:

$$
\begin{aligned}
H_{\mathrm{LSW}}= & \sum_{\mathbf{k} \mu \sigma} E_{\mu}(\mathbf{k}) p_{\mathbf{k}, \mu \sigma}^{\dagger} p_{\mathbf{k}, \mu \sigma}+\sum_{\mathbf{q}} \omega_{\mathbf{q}} \beta_{\mathbf{q}}^{\dagger} \beta_{\mathbf{q}} \\
& +\frac{1}{\sqrt{N}} \sum_{\mathbf{k} \mathbf{q}, \mu \nu \sigma} M_{\mu \nu}(\mathbf{k}, \mathbf{q}) p_{\mathbf{k}-\mathbf{q}, \mu \sigma}^{\dagger} p_{\mathbf{k}, \nu-\sigma}\left(\beta_{\mathbf{q}}^{\dagger}+\beta_{-\mathbf{q}}\right),
\end{aligned}
$$

where $p_{\mathbf{k}, \mu \sigma}^{\dagger}$ represent both $\mathbf{k}$ and $\mathbf{k}+\mathbf{Q}$ states $a_{\mathbf{k}, x \sigma}^{\dagger}, a_{\mathbf{k}, y \sigma}^{\dagger}$, $a_{\mathbf{k}+\mathbf{Q}, x \sigma}^{\dagger}, a_{\mathbf{k}+\mathbf{Q}, y \sigma}^{\dagger}$ for $\mu=1, \ldots, 4$, respectively. $E_{\mu}(\mathbf{k})$ are the eigenvalues of the matrix given by Eq. (22). The bare vertex $M_{\mu \nu}(\mathbf{k}, \mathbf{q})$ has a complex form,

$$
M_{\mu \nu}(\mathbf{k}, \mathbf{q})=\left(u_{\mathbf{q}}+v_{\mathbf{q}}\right) M_{\mu \nu}^{h-s}(\mathbf{k}, \mathbf{q}),
$$

where

$$
\begin{aligned}
M_{\mu \nu}^{h-s}(\mathbf{k}, \mathbf{q})= & \sum_{\xi \xi^{\prime}=1,2}\left[F_{\xi \xi^{\prime}}(\mathbf{k}, \mathbf{q}) V_{\mu \xi}(\mathbf{k}+\mathbf{q}) V_{\nu \xi^{\prime}}(\mathbf{k})\right. \\
& \left.+F_{\xi \xi^{\prime}}(\mathbf{k}+\mathbf{Q}, \mathbf{q}) V_{\mu \xi}(\mathbf{k}+\mathbf{q}+\mathbf{Q}) V_{\nu \xi^{\prime}}(\mathbf{k}+\mathbf{Q})\right]
\end{aligned}
$$

This hole-magnon bare vertex depends on the geometrical factors that follow from the Bogoliubov transformations for fermions $\left[V_{\mu \xi}(\mathbf{k})\right]$ and bosons $\left(u_{\mathbf{q}}, v_{\mathbf{q}}\right)$. Neglecting the $\mathrm{AF}$ corrections to the free Hamiltonian results in the $M_{\mu \nu}^{h-s}(\mathbf{k}, \mathbf{q})$ vertex with the first term in Eq. (27) only. Moreover,

$$
\begin{aligned}
F_{11}(\mathbf{k}, \mathbf{q})= & \left(J_{x, e}+J_{z, e}\right)\left[\cos q_{x}-\cos \left(2 k_{x}-q_{x}\right)\right] \\
- & J_{t}\left[\cos q_{y}-\cos \left(2 k_{y}-q_{y}\right)\right], \\
F_{12}(\mathbf{k}, \mathbf{q})= & 2\left(J_{x, e}-J_{z, e}\right) \sin k_{x} \sin \left(k_{y}-q_{y}\right) \\
& -2 J_{t} \sin k_{y} \sin \left(k_{x}-q_{x}\right),
\end{aligned}
$$

$F_{22}(\mathbf{k}, \mathbf{q})$ and $F_{21}(\mathbf{k}, \mathbf{q})$ are obtained from $F_{11}(\mathbf{k}, \mathbf{q})$ and $F_{12}(\mathbf{k}, \mathbf{q})$ by the transformation $\left(k_{x}, q_{x}\right) \leftrightarrow\left(k_{y}, q_{y}\right)$. At small momenta these factors can be approximated as $F_{\mu \nu}(\mathbf{k}, \mathbf{q})$ $\sim k_{x(y)} \sin q_{x(y)}$ while for large $\mathbf{k} \rightarrow \mathbf{Q}$ they decrease with increasing momentum transfer, $F_{\mu \nu}(\mathbf{k}, \mathbf{q}) \sim \cos q_{x(y)}$. For small momenta the term $u_{\mathbf{q}}+u_{\mathbf{q}} \sim\left(q^{2}-\alpha q_{x} q_{y}\right)^{1 / 4}$, and the vertex $M_{\mu \nu}(\mathbf{k}, \mathbf{q})$ vanishes in the $\mathbf{q} \rightarrow 0$ limit. In the opposite limit, for $\mathbf{q} \rightarrow \mathbf{Q}$ we have $u_{\mathbf{q}}+v_{\mathbf{q}} \sim|\mathbf{q}-\mathbf{Q}|^{-1 / 2}$ but $M_{\mu \nu}^{h-s}(\mathbf{k}, \mathbf{q})$ $\sim|\mathbf{q}-\mathbf{Q}|$ leading to the hole-magnon vertex, $M_{\mu \nu}(\mathbf{k}, \mathbf{q})$ 
$\sim|\mathbf{q}-\mathbf{Q}|^{1 / 2}$, also vanishing in this limit. This behavior of the vertex is different than obtained in the Kondo-lattice model by Ramšak and Prelovšek, ${ }^{26}$ where the form of the Kondo interaction neglects the respective orbital phases in real compounds leading to a diverging vertex for $\mathbf{q} \rightarrow \mathbf{Q}$.

The present model is similar to the one used earlier for spin polarons in the $t-J$ model. ${ }^{7,11,14,27}$ The main differences are another coupling term $H_{h-s}$, as well as the presence of the kinetic terms $\left(H_{h}^{0}, H_{h}^{J}, H_{h}^{A F}\right)$. The latter kinetic parts have a broader dispersion than that found from the $t-t^{\prime}-J$ model for the realistic parameters of high-temperature superconducting oxides, and is thus expected to more strongly influence the respective spectral functions. The Hamiltonian $H_{\mathrm{LSW}}$ also has more independent parameters and can be directly applied to real systems. As in the $t-J$ model, a propagating hole experiences scattering on the spin excitations of the AF background, described by $H_{h-s}$. It has been shown ${ }^{14}$ that an accurate description of the spectral density in the $t-J$ model is obtained by treating these scattering processes in SCBA. ${ }^{7,11}$ A similar quality is expected in the present $4 \times 4$ problem and in the leading order we find the Green function (for $p_{\mathbf{k}, \mu \sigma}$ states)

$$
G_{\mu \nu}^{p}(\mathbf{k}, \omega)=\frac{1}{\omega-E_{\mu}(\mathbf{k}) \delta_{\mu \nu}-\Sigma_{\mu \nu}^{p}(\mathbf{k}, \omega)} .
$$

which is determined in the SCBA by the hole self-energy obtained from the bare vertex and the self-consistently dressed Green function,

$$
\Sigma_{\mu \nu}^{p}(\mathbf{k}, \omega)=\sum_{\alpha \beta, \mathbf{q}} M_{\mu \alpha}(\mathbf{k}, \mathbf{q}) M_{\beta \nu}(\mathbf{k}, \mathbf{q}) G_{\alpha \beta}^{p}\left(\mathbf{k}-\mathbf{q}, \omega-\omega_{\mathbf{q}}\right)
$$

We have solved the system of Eqs. (30) and (29) selfconsistently. Next, we have to project this problem on the states with momentum $\mathbf{k}$ only. Consequently, the Green functions for the original $a_{\mathbf{k}, \nu, \sigma}(\nu=x, y)$ states have been approximated as

$$
G_{\mu \mu}(\mathbf{k}, \omega) \approx \sum_{\nu}\left|V_{\mu \nu}(\mathbf{k})\right|^{2} G_{\nu \nu}^{p}(\mathbf{k}, \omega),
$$

giving the single-hole spectral functions,

$$
A_{\mu \mu}(\mathbf{k}, \omega)=-\frac{1}{\pi} \operatorname{Im} G_{\mu \mu}(\mathbf{k}, \omega+i \epsilon),
$$

exhibiting quite a complex structure that resembles the qualitative results obtained for the $t-J$ (Refs. 9, 12, 14, and 27) and the spin-fermion models. ${ }^{8,26}$

\section{B. Parameters and free-hole dispersion}

We solved the many-body problem given by Eq. (5) using similar parameters as obtained empirically by van Elp et al. ${ }^{28}$ in a $\mathrm{NiO}_{6}$ cluster in octahedral symmetry or in the multiorbital tight-binding model. ${ }^{29}$ We use the following hopping elements between oxygen nearest neighbors: $t_{x x}=t_{y y}$ $=0.20, t_{x y}=t_{y x}=-0.35 \mathrm{eV}$, and between $2 p$ and $3 d$ orbitals at nearest neighbors: $t_{x}=1.5, t_{z}=t_{x} / \sqrt{3}$, and $t_{\pi}$ $=1.0 \mathrm{eV}$. The Coulomb interaction elements are $U\left({ }^{4} T\right)$ $=6.7+10 D_{q}, U\left({ }^{2} T\right)=9.1+10 D_{q}$, and $U\left({ }^{2} E\right)=9.1$, while
$\Delta=5.0$ and the $e_{g}-t_{2 g}$ splitting is $10 D_{q}=0.7$ (all in $\mathrm{eV}$ ). This gives the three-site hoppings: $T_{x, e} \simeq-0.10, T_{z, e} \simeq-0.03$, $T_{t} \simeq-0.31$, and Kondo interactions $J_{x, e} \simeq 0.52, J_{z, e} \simeq 0.17$, $J_{t} \simeq-0.06$ (all in eV). Thus, the model is dominated by an AF coupling with the $e_{g}$ symmetry, accompanied by a small FM coupling for the $t_{2 g}$ states.

All the numerical calculations were carried out on a $20 \times 20$ cluster with toroidal boundary conditions in the irreducible wedge of the unfolded BZ. The energy axis was divided into 2000 points with resolution $\delta \omega \sim 0.01 \mathrm{eV}$, and we stopped iterating when the changes in $\Sigma_{\mathbf{k}, \omega}[A(\mathbf{k}, \omega)]^{2}$ became less than $0.1-0.01 \%$ (this corresponds to $20-70$ iterations, depending on the value of $J$ ).

The superexchange parameter can be estimated using the fourth-order expression

$$
J=\frac{2\left(t_{x}^{2}+t_{z}^{2}\right)^{2}}{\Delta^{2}}\left[\frac{1}{U\left({ }^{2} E\right)}+\frac{1}{\Delta}\right]
$$

yielding $J \approx 0.072 \mathrm{eV}$. Although the above expression predicts a reasonable magnitude of $J$, it might be still underestimated as there are also significant fifth-order contributions, due to the oxygen-oxygen hoppings, similar to those considered by Eskes and Jefferson for $\mathrm{CuO}_{2}$ planes. ${ }^{30}$ The actual value of $J$ does not change the electronic states in a significant way, and we have chosen $J=0.1 \mathrm{eV}$ as a representative value. Furthermore, we took the unimportant FM exchange interaction $\left(J^{\prime}\right)$ between nearest-neighbor $\mathrm{Ni}$ ions to be smaller by one order of magnitude than $J \quad(\alpha=0.1)$.

The noninteracting ( $U=J_{H}=0$, etc.) version of the above model, using the parameters of van Elp et al. ${ }^{28}$ yields a satisfying fit to the (unpolarized) LDA band structure. ${ }^{4,5}$ This is not entirely unproblematic since the band structure of a thin 2D slab is compared with that of the fully three-dimensional system. It turns out, however, that the bands derived from the planar $2 p$ orbitals map quite well on the LDA bands of the same character, at least as long as $k_{z}=0$ and the only major difference is in our neglect of the out-of-plane $p_{z}$ orbitals. The band that involves these states is degenerate with one of the planar bands along the $\langle 01\rangle$ direction, but the most dispersive feature in the $\langle 11\rangle$ direction (Fig. 15 of Ref. 5) turns out to be of $p_{z}$ character. We suspect that this band can be better neglected because it is most strongly affected by the loss of $k_{z}$ as a good quantum number. More generally, on the level of the spin-hole dynamics we expect that no complications occur because of our neglect of the third direction. The reason is that it mainly involves magnons capable of creating a spin backflow opposite to the momentum of the hole. ${ }^{10}$

In a $2 \mathrm{D}$ model of $\mathrm{NiO}$ one finds two oxygen bands described by $H_{h}^{0}$, with a dispersion of about $3 \mathrm{eV}$. This dispersion is shown in Fig. 2(a) along the main directions within a 2D Brillouin zone (Fig. 1). The bands are degenerate along the $\Gamma-M$ direction. The zeroth-order coupling to the correlated $\mathrm{Ni}(3 d)$ orbitals gives the effective hopping via $\mathrm{Ni}$ sites, which modifies strongly these hole dispersions and, as a result, both oxygen bands $\left(H_{h}^{0}+H_{h}^{J}\right)$ get much closer to each other [solid lines in Fig. 2(b)]. In the $\Gamma-X(\Gamma-Y)$ direction they are strongly influenced by $H_{h}^{J}$, while along the $\Gamma-M$ direction the dispersions are only slightly pushed upwards. The largest effective hopping is in the $t_{2 g}$ channel leading to 

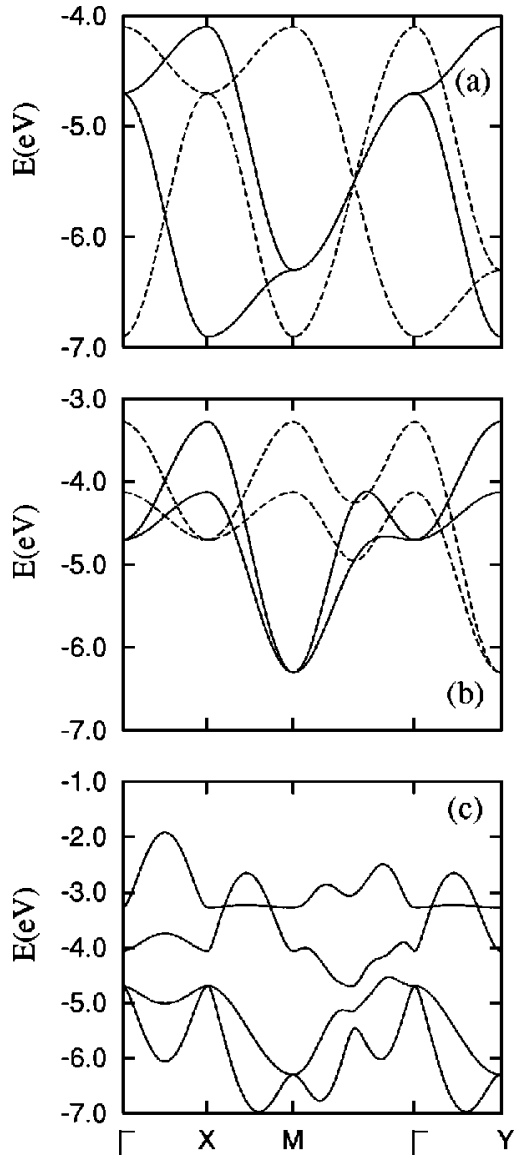

FIG. 2. The oxygen electron bands as obtained from the free and effective hopping terms: (a) $H_{h}^{0}$, (b) $H_{h}^{0}+H_{h}^{J}$, and (c) from the total free Hamiltonian for oxygen electrons, $H_{h}^{0}+H_{h}^{J}+H_{h}^{A F}$, for realistic parameters of $\mathrm{NiO}$. Solid (dashed) lines in (a) and (b) represent states for momentum $\mathbf{k}(\mathbf{k}+\mathbf{Q})$. In (c) bands are coupled by the nesting vector $\mathbf{Q}$ (see Fig. 1) and cannot be labeled by individual momenta $\mathbf{k}$ and $\mathbf{k}+\mathbf{Q}$.

strong renormalization for the lower band, which is of this character. Including the $H_{h}^{A F}$ term, one finds that the oxygen bands at a given momentum $\mathbf{k}$ mix with the ones for $\mathbf{k}+\mathbf{Q}$ [see Fig. 2(c)] resulting in a spectrum broader by $\sim 2 \mathrm{eV}$ more than that found for $H_{h}^{0}+H_{h}^{J}$ alone [Fig. 2(c)]. Moreover, the $\langle 11\rangle$ and $\langle\overline{1} 1\rangle$ directions are no longer equivalent. These bare dispersions have little in common with either the LDA-band structure or with the experiment. ${ }^{5}$

For a comparison, the truly noninteracting tight-binding band structure is presented in Fig. 3. Here we have included all $p$ and $d$ states for the (001) plane in the $U, J_{H} \rightarrow 0$ limit. The CT energy between $\mathrm{O}$ and $\mathrm{Ni}$ states $\Delta=2.0 \mathrm{eV}$ was chosen to give realistic splittings between the $p$ and $d$ bands. ${ }^{31}$ We find three Ni bands above the two bands having predominantly oxygen character. The $p$-like bands are similar to those obtained by Shen et al. within LDA. ${ }^{5}$

The bands from Fig. 2(c) do not contribute equally to a given $\mathbf{k}$ state. Thus, we have calculated the spectral functions $A_{\mu \mu}^{0}(\mathbf{k}, \omega)$ obtained in the limit $M_{\mu \nu}(\mathbf{k}, \mathbf{q})=0$ (no coupling to magnons). ${ }^{32}$ At the high-symmetry points (where $H_{h}^{A F}$ part of the free Hamiltonian does not contribute) we have found one (at the $\Gamma$ and $M$ points) or two (at the $X$ and $Y$ points) peaks representing the bands from Fig. 2(b). The spectra are

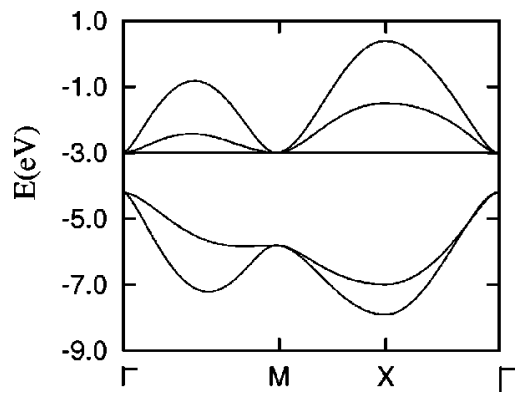

FIG. 3. Five-band electronic structure of $\mathrm{NiO}$ as obtained from the realistic multiband model (including $2 p$ and $3 d$ orbitals) in the $U \rightarrow 0$ limit, assuming $\varepsilon_{d}=-3.0 \mathrm{eV}$ and $\Delta=2.0 \mathrm{eV}$.

more complex at intermediate momenta where all four bands from Fig. 2(c) contribute (with different spectral weights) to $A_{\mu \mu}^{0}(\mathbf{k}, \omega)$ (see Fig. 4). However, these spectra, with four or eight (in the $\langle 11\rangle /\langle\overline{1} 1\rangle$ direction) dispersive features, look completely different than the photoemission data by Shen et al. ${ }^{5}$ As we will show below, this difference is removed when the states are dressed by the coupling of a moving hole to the quantum fluctuations in spin background.

\section{NUMERICAL RESULTS AND DISCUSSION}

\section{A. Spectral functions for a spin-fermion model}

In Figs. 5 and 6 we show our results for the spectral functions as line plots along high-symmetry directions in momentum space. They are drastically modified with respect to those discussed above in the absence of hole-spin coupling, and are now characterized by flat features at low energies accompanied by dispersive features at high energies; the latter are well visible on the incoherent background.

In order to arrive at a meaningful comparison with experiment, one should keep in mind that it is impossible to address the spectral weight distributions of the photoemission
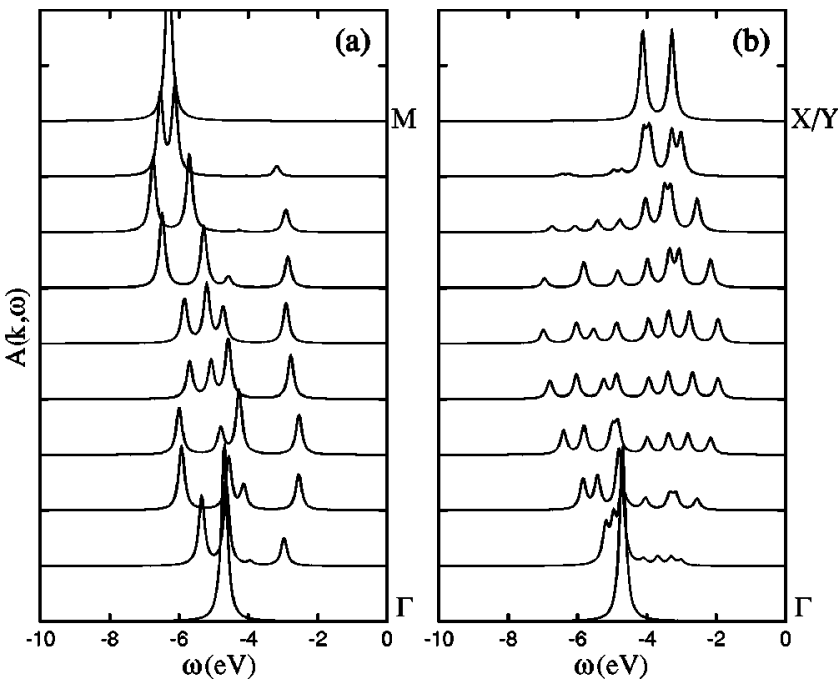

FIG. 4. The electronic spectral functions for the total free Hamiltonian, $A_{\mu \mu}^{0}(\mathbf{k}, \omega)$, as obtained using realistic parameters along (a) $\Gamma-M$ [the $\langle 100\rangle$ direction by Shen et al. (Ref. 5)], and (b) the combination of $\Gamma-X$ and $\Gamma-Y$ directions $[\langle 110\rangle$ direction by Shen et al. (Ref. 5)], respectively. The large broadening $\epsilon$ $=0.1 \mathrm{eV}$ is used. 


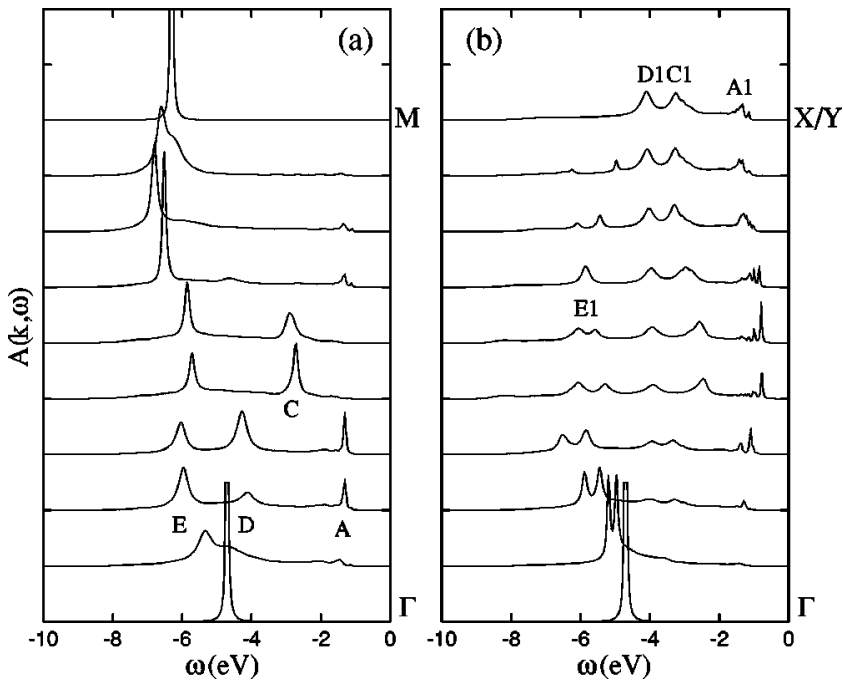

FIG. 5. The electronic spectral functions in the SCBA, $A_{\mu \mu}(\mathbf{k}, \omega)$, for the realistic parameters of $\mathrm{NiO}$ along: (a) $\Gamma-M$ direction [the $\langle 100\rangle$ direction by Shen et al. (Ref. 5)], and (b) the combination of $\Gamma-X$ and $\Gamma-Y$ directions $[\langle 110\rangle$ direction by Shen $e t$ al. (Ref. 5)], respectively. The broadening $\epsilon=0.01 \mathrm{eV}$ is used.

spectra in full quantitative detail. The problem is on the experimental side; photoemission does not directly measure the single-hole spectral function, but instead a related quantity that is modulated by dipole matrix element effects, photoelectron (final) state effects, etc. ${ }^{33}$ These are known to reshuffle the spectral weights considerably. Special to the present case, we have calculated the $\mathrm{O}(2 p)$ spectral weights, while in experiment both $2 p$ and $3 d$ weights are measured. As will be further explained, this causes large differences in the intensities of the low-lying states.

Having this in mind, let us compare the overall appearance of our central results (as shown in Fig. 5) with experiment. ${ }^{5}$ Figure 5(a) (with spectra along the $\Gamma-M$ direction) can be directly compared with the data along the $\langle 100\rangle$

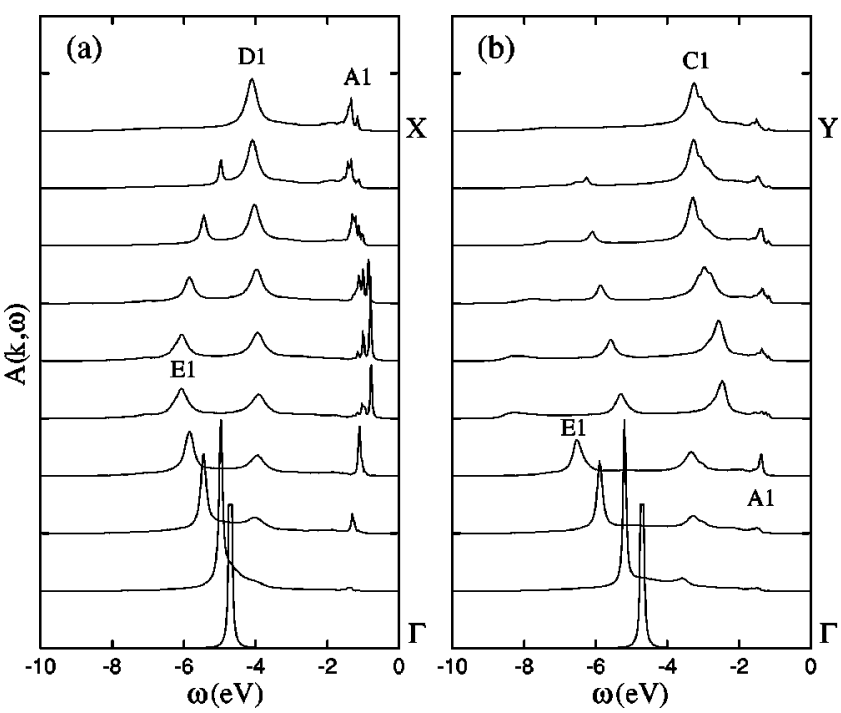

FIG. 6. The electronic spectral functions in the SCBA, $A_{\mu \mu}(\mathbf{k}, \omega)$, for the realistic parameters of $\mathrm{NiO}$ along the (a) $\Gamma-X$ and (b) $\Gamma-Y$ direction, respectively. The broadening $\epsilon=0.01 \mathrm{eV}$ is used. direction (Figs. 2-13 in Ref. 5). Shen et al. argue that the experiment most likely averages over the magnetically inequivalent $\Gamma-X$ and $\Gamma-Y$ directions, and for this reason we show in Fig. 5(b) the superposition of the spectral functions in these two directions, to be compared with the " $\langle 110\rangle$ " results of Shen et al. (their Figs. 14-22). The spectral functions resolved along the nonequivalent $\Gamma-X$ and $\Gamma-Y$ directions are shown in Figs. 6(a) and 6(b) separately.

As a somewhat unexpected result, Shen et al. reported that the spectral functions along the $\langle 100\rangle$ and $\langle 110\rangle$ directions look quite similar. ${ }^{5}$ Taking all information together, there seem to be four distinguishable features: $A, C, D$, and $E$ along $\langle 100\rangle$, and $A 1, C 1, D 1$, and $E 1$ along the $\langle 110\rangle$ direction, taking the notation used in Ref. 5. These fall in two groups: two low-lying features $(A / A 1, C / C 1)$ showing very little (if any) dispersion, and two "bandlike" features $(D / D 1, E / E 1)$ at higher energies, where especially $E / E 1$ seems to show sizable dispersion at larger momenta.

At intermediate energies $(-6 \mathrm{eV}<\omega<-4 \mathrm{eV})$ and away from the $\Gamma$ point, some of the pronounced bands in the bare functions (Fig. 4) cannot be identified in our spectra (Fig. 5). This difference is due to the spin fluctuations that are building up low-energy coherent states, and at the same time wipe out some of the bare bands leaving only the highenergy sector coming from the bonding band from Fig. 2. As a result, our spectra can be, therefore, divided into (i) bound states ( $A / A 1$ features and to some extent $C / C 1$ features), (ii) nonbinding band states at intermediate energies, except for the special momenta where the hole-magnon vertex becomes small (most of $D / D 1$ and $E / E 1$ features), and (iii) antibound states (tiny states at $\omega \simeq-8 \mathrm{eV}$ seen in the $\Gamma Y$ direction). The most spectacular example of these effective states is found along the $\langle 11\rangle /\langle\overline{1} 1\rangle$ direction where eight bands [Fig. 4(b)] are reduced in the self-consistent procedure to at most five features [Fig. 5(b)].

On a more detailed level one might argue that at least superficially there appear to be discrepancies. However, further analysis shows a definite meaning to these discrepancies, some of which might hopefully stimulate further experimental investigation. We address this problem systematically for different experimentally observed features.

\section{B. Bound states and their momentum dependencies}

Let us first focus on the low-energy dispersionless states $A / A 1$ and $C / C 1$. According to our calculation, these are bound states appearing above the top of the bare $2 p$ band, and they only exist in the self-consistent theory. They can be looked at as local doublets, in analogy to the ZR singlets in the cuprates; ${ }^{6}$ their wave functions describe $2 p$ holes bound to spin flips. In the entirely localized case, this would invoke a single localized spin flip: the doublet with $M_{t o t}=1 / 2$, which can be written as $|\Psi\rangle \propto(\sqrt{2}|1,-1 / 2\rangle-|0,1 / 2\rangle)$, where $|M, m\rangle$ refers to the $z$ th component $|M\rangle$ of $S=1 \mathrm{Ni}$ spin, and to the $z$ th component $|m\rangle$ of the $2 p$ hole with spin $s=1 / 2$, localized in a wave packet centered around this particular $\mathrm{Ni}$ site. $|1,-1 / 2\rangle$ can be taken to be the classical reference state (free hole and Néel vacuum), and $|0,1 / 2\rangle$ corresponds then with the local spin flip responsible for the binding. The wave function in SCBA is more involved, however. In addition to these local spin flips it also includes a large number of spin 


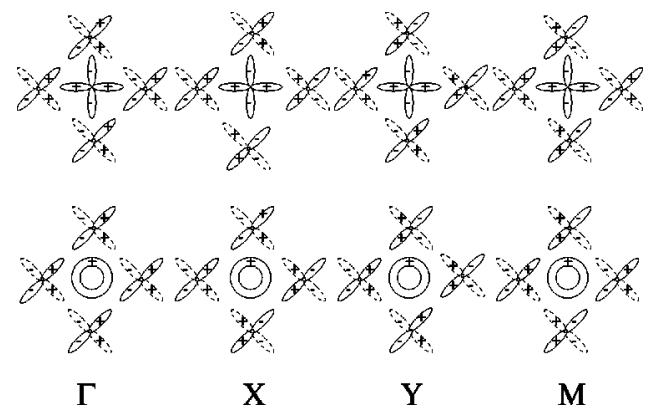

FIG. 7. The phases of $\mathrm{O}(2 p)$ orbitals with regard to $x^{2}-y^{2}$ (top) and $3 z^{2}-r^{2}$ (bottom) Ni orbitals at various high-symmetry points in momentum space.

flips associated with the local quantum disorder in the spin system, caused by the short-range delocalization of the hole, similarly as to what is found in the $t-J$ model. ${ }^{10}$ Starting with the spin-fermion model, it is therefore possible to arrive at a unifying treatment of the formation of the local bound states and their subsequent $t$ - $J$-like delocalization.

As it turns out, the formation of the bound states is easier to understand in the $\Gamma-X$ and $\Gamma-Y$ directions, and we first focus on these directions, setting the stage for what is going on in the $\Gamma-M$ direction. The features $A 1$ and $C 1$ are bound states, finding their origin in the antibonding $\mathrm{p}$ bands of the noninteracting band structure. It is actually the case that the $A 1$ feature is best developed at the $X / 2$ point [Fig. 6(a)], while the much broader $C 1$ feature develops at all $\mathbf{k}$ values along the $\Gamma-Y$ direction [Fig. 6(b)]. Using symmetry arguments as explained in more detail in the Appendix, this allows us to identify precisely the microscopic nature of these states. Because of our strong coupling assumption, the $3 d$ states have to be considered as localized while the (bare) $2 p$ states are the band states with particular momenta. To find out the magnitude of the spin-hole coupling, we have to project the band states at a given momentum on the irreducible representations of the $D_{4 h}$ point group symmetry at $\mathrm{Ni}$ sites. Only at the high-symmetry points $(\Gamma, X / Y, M)$ do these band states correspond with precise irreducible representations of the local symmetry group. At the $\Gamma$ point the phases of the $2 p$ orbitals can be taken as indicated in Fig. 7. By multiplying these with the phase factors $\exp (i \mathbf{k} \cdot \mathbf{R})$, the phases of $2 p$ orbitals at $X / Y, M$ points follow as in Fig. 7. However, for $t_{p p} \neq 0$ the oxygen orbitals are no longer orthogonal and one should consider instead the wave functions rotated by $\pi / 4$ in the (001) plane to obtain the appropriate meaning of both spectral functions.

Because of its kinetic origin, the strength of the Kondo coupling can be directly determined from the strength of the bare hybridization. If the local $d$ state is nonbonding with regard to the band state, the Kondo coupling vanishes, while a maximal hybridization implies as well a maximal holemagnon scattering. It is directly seen from Fig. 7 that the bare hybridization to the $x^{2}-y^{2}$ and $3 z^{2}-r^{2}$ states vanishes both at the $\Gamma$ and the $M$ point; this explains why the lowenergy bound-state structures are missing at these points [see Fig. 5(a)]. Moreover at the $X$ point one can expect the strongest bound states in the $x^{2}-y^{2}$ channel. However, the magnetic umklapp $\mathbf{k} \rightarrow \mathbf{k}+\mathbf{Q}$ scattering leads to the strongest $\mathrm{QP}$ at the $X / 2$ point in this channel. The conclusion is obvious: the Al feature is associated with the $x^{2}-y^{2}$ local doublet. The maximum of this bound state at the $X / 2$ point rather than at the $X$ point is similar to our recent findings in the spinfermion model for the $\mathrm{CuO}_{2}$ plane where pronounced bound states build up at intermediate momenta close to the $(\pi / 2, \pi / 2)$ point $^{34}$ in agreement with the photoemission experiments, ${ }^{35}$ although from a simple symmetry consideration one would expect it to be best developed at the $(\pi, \pi)$ point.

Let us now consider the $3 z^{2}-r^{2}$ sector. It follows from Fig. 7 that the coupling is now at maximum at the $Y$ point. Therefore, we conclude that the $C 1$ feature corresponds with the $3 z^{2}-r^{2} \mathrm{ZR}$ doublet. This state is at higher energy because the Kondo coupling ${ }^{18}$ involving the $3 z^{2}-r^{2}$ state is weaker than in the $x^{2}-y^{2}$ case. The coupling is proportional to the hopping matrix element squared, and the in-plane $p-d$ hopping is by a factor of $1 / \sqrt{3}$ less for $3 z^{2}-r^{2}$ orbitals than for $x^{2}-y^{2}$ orbitals. ${ }^{24}$ Hence, the Kondo coupling is weaker by a factor of 3 and this barely suffices to generate bound states in the $3 z^{2}-r^{2}$ sector, while the doublets that involve the $x^{2}-y^{2}$ states are strongly bound. This can be seen in Fig. 2 (c) where, e.g., the bands crossing along the $\Gamma-X$ direction [Fig. 2(b)] are separated into two highly dispersive (bondingantibonding) states and the other two states with small dispersion. Moreover, in the $x y$ sector the coupling is also the strongest at the $Y$ point resulting in the less bound part of the broad feature $C 1$ that consists of more than one distinguishable part [see Fig. 6(b)]. The other state with $3 z^{2}-r^{2}$ symmetry, although mixed with $x y$ states, is the $D 1$ feature in the $\langle 11\rangle$ direction [see Fig. 6(a)]. The nonbonding character of this state is consistent with its location at intermediate energies.

Coming back to the experiment, ${ }^{5}$ we find that the energetics of the calculated $A / A 1$ and $C / C 1$ features is roughly correct, while the intensities are quite different from the experiment. For instance, in the experiment the $A / A 1$ and $C / C 1$ peaks dominate at the $\Gamma$ point, while we find there very small spectral weights. Worse, at first sight it might appear as rather odd to indicate the $A$ feature in Fig. 5(a) although it is clearly distinguishable from the background only in a very narrow momentum range. By considering the symmetry arguments it is clear, however, why the lowestenergy $A$ feature is scarcely seen along the $\langle 10\rangle$ direction in the calculation. The hybridization with the $x^{2}-y^{2}$ states vanishes at the $\Gamma$ and $M$ points. Although it becomes finite away from the end points so that the $A$ feature becomes visible at intermediate momenta, it is at best rather weak. Similarly, the hole-magnon coupling in the $3 z^{2}-r^{2}$ and $x y$ channels is also weaker in the $\Gamma-M$ direction as compared to the $\Gamma-X / Y$ directions, and for the same reasons as for the $A$ peak, the $C$ feature is not very pronounced along $\Gamma-M$.

Let us address the behavior of the ZR states at small momenta. From Fig. 4 it is seen that the calculation without the hole-magnon coupling predicts a single delta function at the $\Gamma$ and $M$ points (the width comes from the artificial broadening), signaling the vanishing of hole-magnon coupling at this momentum. Let us consider the limiting case that the Kondo exchange is much larger than the $2 p$ bandwidth, while the Heisenberg exchange $J$ vanishes. It is easy to see that in this limit a (tightly bound) ZR doublet will be formed 
that is localized around one particular Ni site. This means that this state exists at all momenta. Nevertheless, because of the disappearance of the bare vertex it would be hardly visible in the $p$-spectral function at the $\Gamma$ point. As shown in the three-band Hubbard model for the cuprates, ${ }^{36}$ the $d$-like spectral function dominates in the low-energy sector near the $\Gamma$ point. We remind the reader that in the context of the cuprates the existence of a ZR state at the $\Gamma$ point has been the subject of considerable debate. ${ }^{37}$

Figure 6 shows that the $A 1$ feature is characterized by a small dispersion in the $\Gamma-X / Y$ directions $(E \simeq 0.2 \mathrm{eV})$ which is similar to the $t-J$ model: the bandwidth is close to $J z S$, a number expected when this bandwidth would be entirely due to quantum spin fluctuations. This is at first sight a surprising outcome, since the spin-fermion model is characterized by a large bare kinetic energy ( $\mathrm{O} 2 p$ bandwidth) which is missing in the $t-J$ model. It can, however, be understood in terms of the ZR localization: also in our theory the binding of the O $2 p$ hole to a particular Ni spin can be regarded as complete and at long times the QP propagates as the hole in the $t-J$ model, mediated by the spin fluctuations in the background. The rather dispersionless appearance of the lowenergy states in the experiment ${ }^{5}$ is therefore not inconsistent with our findings. We notice, however, that there is convincing evidence, both theoretically ${ }^{38}$ and experimentally, ${ }^{39}$ for the importance of lattice driven self-localization in nickelates. Another explanation for the lack of dispersions could therefore be the electron-phonon coupling leading to small polaron formation. The essential difference between phonon self-localization and the spin-driven affair discussed here is that in contrast to the latter, the phonon mechanism involves, in the first instance, optic modes. Accordingly, it is characterized by much less momentum dependence than the spin case, and the mechanism giving rise to the QP bandwidth in the magnon case is basically absent when phonons are involved.

It should also be noticed that the rather good comparison of the calculated $A-C$ splitting with experiment is actually rather accidental. We consider a single atom layer slab, while in reality this slab is connected with a half-infinity of bulk, which will surely renormalize the splitting. Obviously, it is unreasonable to expect that a simple model as we are using can be quantitatively accurate in this regard.

To sum up, the symmetry-dictated momentum dependencies of the intensities of the $A / A 1$ and $C / C 1$ features should be considered as the main result of this paper and its predecessor. ${ }^{17}$ In principle, it allows for a further empirical characterization of the nature of these states. Within our theorist's limitation, we believe it should be possible to design experiments specifically targeting this issue. The strategy could be to tune photon energies such that the $2 p$ spectral weights are enhanced over the $3 d$ weights and look for relative changes in the intensities. These should reflect the extreme momentum dependence as we find in our theoretical results. For instance, the weight in the low-energy features should get drastically reduced at the $\Gamma$ point. ${ }^{36}$ Is there any ground to believe these effects on the basis of existing data? It is striking that the $A$ feature weakens approaching the high and small momenta points along the $\langle 100\rangle$ direction in the experiment of Shen et al. ${ }^{5}$ (Figs. 7 and 8 in Ref. 5), while it is much stronger along the $\langle 110\rangle$ direction (Figs. 14, 19, and 20 in Ref. 5). This is even in semiquantitative agreement with our analysis.

To end this section, let us discuss the precise status of the quasiselection rule governing the extreme momentum dependences of the intensities in our calculation. These selection rules are actually not robust-they are only exactly obeyed in the strong coupling limit: $U / t, \Delta / t,(U-\Delta) / t \rightarrow \infty$, which is straightforward to see. In the argument involving Fig. 7, we insisted on the locality of the $d$ states. As can be seen by comparing Fig. 2(a) with Fig. 3, this works quite differently when the $d$ states are treated as Bloch states. The $3 d$-Bloch states hybridize with the $2 p$ states at all momenta, with the exception of the $\Gamma$ and $M$ point. At the $\Gamma$ point, the $2 p$ states at $\sim-4.7 \mathrm{eV}$ (Fig. 2) are thus not shifted by the hybridization with the $3 d$ states (Fig. 5). On the contrary, both at the $X$ and $Y$ points it is seen that the $2 p$ states are shifted downwards due to the $p-d$ hybridization. At the $M$ point only the $p-p$ hybridization is responsible for the lower energy of the QP state in contrast to the $\Gamma$ point.

As we already explained in the Introduction, $\mathrm{NiO}$ is in the "intermediate regime" of the old Zaanen-Sawatzky-Allen classification scheme. ${ }^{19}$ This was the conceptual novelty in this paper: although both $\Delta / t$ and $U / t$ are large, $(U-\Delta) / t$ is small with the consequence that the hole dynamics is a lowdensity analogue of the mixed valence regime. Compared to the strong-coupling limit considered here, the charge dynamics has to be considerably softer in reality. In the light of the general experience, ${ }^{40}$ it is expected that this softening manifests itself in first instance in the spectral weight redistributions. Since the selection rules are in some sense an artifact of the strong-coupling limit, the first manifestation of the softening of the charge dynamics has to be that spectral weight is transferred from the high-lying incoherent $2 p$-like states into the near-threshold ZR sector. This is the reason that we do not hesitate to claim an $A / C$ feature along $\Gamma-M$, although it does not have much spectral weight in the calculation.

\section{Umklapp hole-magnon scattering}

Consider the motion of the hole in the real space corresponding with momenta along the $\Gamma-X$ and $\Gamma-Y$ directions, respectively. On the one hand, from Fig. 1 it is seen that the hole with momentum along the $\Gamma-X$ direction moves from one sublattice to the other. On the static level (no spin waves) this causes a severe magnetic umklapp scattering at the magnetic zone boundary (Fig. 2). On the other hand, on the static level the hole with momentum along the $\Gamma-Y$ direction moves on a parallel spin background, and there is no umklapp in this direction. The absence of a magnetic gap on the classical level has the effect that the bare kinetic energy is much larger, as is evident from Fig. 2. Let us now consider the dynamical theory. One could be tempted to think that since the $\Gamma-Y$ hole moves effectively through a ferromagnet, a hole of this kind would not scatter against spin waves at all. Considering Fig. 6 one finds that this is not the case. Especially at larger momenta the spectral function bears little resemblance with the free-particle spectrum and the holemagnon scattering is strong. 
It can be checked that if the static umklapp scattering would be neglected, the hole-magnon scattering would be identical along the $\Gamma-X$ and $\Gamma-Y$ directions (see Ref. 17). Although the inclusion of the umklapp changes the detailed momentum dependencies of the bare vertex [Eq. (27)], its average strength is barely affected. The rather different appearance of the spectral functions along the $\Gamma-X$ and $\Gamma-Y$ directions is therefore primarily caused by the decrease of free band width in the "antiferromagnetic" $\Gamma-X$ direction (Fig. 2). In other words, the umklapp scattering leads to the $Y / 2$ point being nonbonding (and to hardly visible $A 1$ feature at this point) while at the $X / 2$ point a strong QP $A 1$ state is observed. Thus, we have to conclude that the magnetic umklapp scattering is important and has to be included if a quantitative analysis of the spectral functions is made. ${ }^{18,41} \mathrm{In}$ fact, the overall shape of the bound-type spectral function at the $X / 2$ point [Fig. 6(a)] starts to resemble what is found in the $t-J$ model, where the free kinetic energy is entirely absent.

\section{Band and scattering states at higher energies}

As we have emphasized in the Introduction, the presence of the dispersionless states at low energies in the one-hole spectral function is consistent with the expectations following from the experience with bound states in the cuprates. The surprise comes due to the simultaneous observation of the strongly dispersive features $D / D 1$ and $E / E 1$ at high energy. ${ }^{5}$

In fact, in our calculations the dispersive high-energy features (along the $\Gamma-X$ and $\Gamma-Y$ directions) are quite well reproduced. In first instance these can be looked at as slightly shifted and broadened versions of the lowest-lying band states of Fig. 4 [notice that in Fig. 4(a) much larger broadening $\epsilon=0.1$ was used than assumed in Figs. 5 and 6, where $\epsilon=0.01]$. This is quite literally true for the sharp feature $D 1$ that is quite distinct in the $\Gamma-X$ direction [Fig. 6(a)]. This feature turns out to be the $3 z^{2}-r^{2}$ symmetry state, with the energy in the vicinity of the noninteracting band state. In the $\Gamma-Y$ direction the broad feature $E 1$ is also well visible at small momenta. In the experiment, the $E / E 1$ states are most convincing as "bandlike states."

That these dispersive features appear in this rather welldefined manner should be regarded as a quantitative matter for which an explicit computation is needed. The only qualitative statement that can be made is that at certain momenta the hole-magnon vertex vanishes, as explained in the previous section. Obviously, at these special momenta the $2 p$ holes are not affected by the scattering against the spin waves. Away from these momenta it is about the relative strength of the hole-magnon scattering in the various bare bands and this is a complicated matter. The main quantitative outcome of our calculations is that apparently the holemagnon coupling is at the same time strong enough to cause a strong ZR localization while certain high-energy $2 p$ electrons scatter off magnons sufficiently weakly such that these states can be understood in terms of bare band states dressed up with weak self-energy effects.

Although we already argued that the gross, qualitative comparison is quite convincing, one might be more skeptical about the detailed comparison, especially regarding the

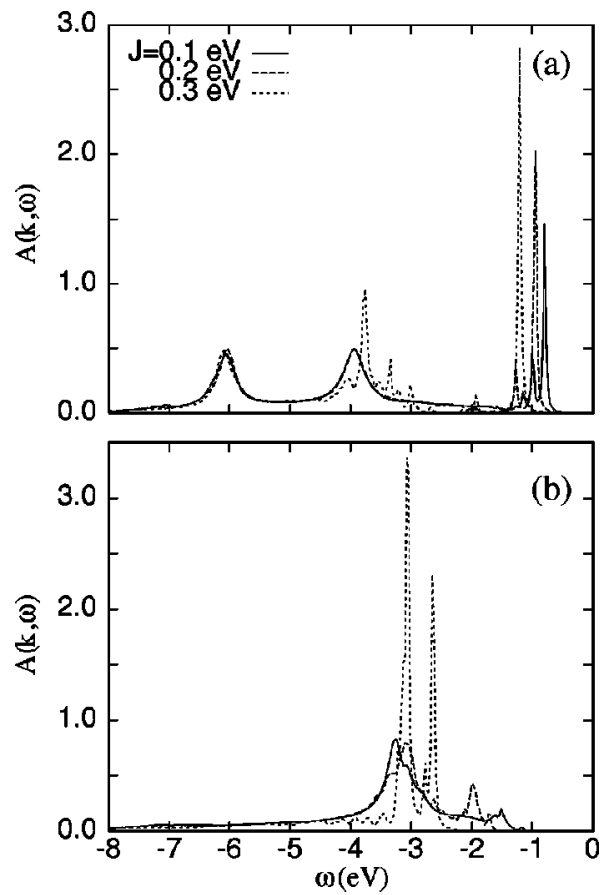

FIG. 8. The electronic spectral functions $A(\mathbf{k}, \omega)$ for the realistic parameters of $\mathrm{NiO}$ at (a) the $X / 2$ point, and (b) the $Y$ point, as obtained for $J=0.1,0.2$, and $0.5 \mathrm{eV}$.

"band-like" features. The problem is that the quantitative accuracy of the bare model is much more directly probed at these higher energies than is the case of the low-energy sector that is dominated to such an extent by the interaction effects that the fine details of the bare model are wiped out. The bare model is of course fairly crude. It is limited to a slab, and, moreover, it neglects many details of the bareband structure, like $s-p$ hybridization, longer-range $p-p$ hoppings, etc., which are known to give rise to sizable distortions of the bare band structure. In this regard, the $\Gamma$ and $M$ points have a special status because of the aforementioned decoupling of the bare band from the magnons.

\section{E. Results for different superexchange and Kondo elements}

Finally, we present the dependence of such characteristic QP features like spectral weights and dispersions on the superexchange interaction, and compare our numerical results with those known for the $t-J$ model. ${ }^{11,14}$ The dependence of the QP states on the value of superexchange between localized spins $J$ (for a fixed ratio $J^{\prime} / J=0.1$ ) is shown in Fig. 8 . At the $X / 2$ point one finds a decrease in the binding energy with increasing superexchange energy, with some secondary bound state above the main ZR peak, while the high-energy states at $\omega \sim-6.5 \mathrm{eV}$ are hardly changed by the scale of spin fluctuations, supporting their interpretation as bandlike oxygen states [see Fig. 8(a)]. The total energetic extent of the spectra remains almost not effected by the magnon energetic scale. ${ }^{42}$ At the $Y$ point the spectra for larger $J$ develop irregular structure at intermediate energies [see Fig. 8(b)], showing that it is difficult to form a QP state in this case due to the umklapp scattering.

In Table I we present the spectral weights and binding energies (relative positions of QP's to the free oxygen bands) 
TABLE I. QP spectral weights $a_{\mathbf{k}}$ and positions of the coherent states relative to the free oxygen band $E_{b}$ (in $\mathrm{eV}$ ) at the $X / 2$ and $X$ points for various values of $J$ (in $\mathrm{eV}$ ) as obtained for the realistic parameters.

\begin{tabular}{ccccc}
\hline \hline & \multicolumn{2}{c}{$\mathbf{k}=(\pi / 4, \pi / 4)$} & \multicolumn{2}{c}{$\mathbf{k}=(\pi / 2, \pi / 2)$} \\
$J$ & $a_{\mathbf{k}}$ & $E_{b}$ & $a_{\mathbf{k}}$ & $E_{b}$ \\
\hline 0.1 & 0.106 & 1.14 & 0.026 & 2.13 \\
0.2 & 0.152 & 0.96 & 0.145 & 1.68 \\
0.5 & 0.208 & 0.75 & 0.331 & 1.14 \\
\hline \hline
\end{tabular}

at the $X / 2$ and $X$ points in the BZ of Fig. 1. One finds that the QP weight grows up, absorbing gradually the incoherent part with increasing $J(\alpha=0.1)$, while the binding energy $\left(E_{b}\right)$ decreases. The strong QP's at the $X / 2$ point occur quite close to the free band, but the spectral weight does not exceed $\sim 0.2$ even at $J=0.5 \mathrm{eV}$, as the free spectrum at this point consists of four peaks [see Fig. 4(a)], and only the lowest one plays an active role in the formation of the bound state. Therefore, the strength of the QP state at the $Y$ point, with only two peaks in the free spectrum, exceeds the one at the $X / 2$ point for large values of $J$.

\section{SUMMARY AND CONCLUSIONS}

Our results demonstrate that the essential features observed in the photoemission for $\mathrm{NiO}$ may be understood in terms of a strongly correlated spin-fermion model. The dispersionless states $(A / A 1, C / C 1)$ together with strongly dispersive ones $(D / D 1, E / E 1)$ have been found and identified with features seen in the photoemission experiments. ${ }^{5}$ As we discussed, the bound states have predominantly $x^{2}-y^{2}$ symmetry, while the other two channels form mainly the strongly dispersive band part of the spectra at intermediate energies. Therefore, this work shows that proper treatment of both "localized" and "itinerant" states is essential in MottHubbard systems, while both the cluster and impurity model approaches, and the conventional band-structure theory, miss this important aspect.

The quantum fluctuations of the background antiferromagnet play a prominent role in causing spectral functions showing both localized and dispersive features: a different spectral function is obtained when the Ising limit is considered instead, resembling the $t-J_{z}$ model. However, in order to obtain the ladder spectrum within the SCBA, in analogy to the $t-J_{z}$ model, ${ }^{14}$ one needs to make further simplifying assumptions for the hopping elements in the present spinfermion model. ${ }^{43}$

The spin-fermion model as derived by us was obtained using perturbation theory in the strongly correlated regime. This implies certain limitations, as we had to assume that $t_{p d} \ll U-\Delta$, and the oxygen $p$ states are well separated from all $d^{7}$ configurations. In reality the lowest $d^{7}\left({ }^{2} T\right)$ configuration is strongly hybridized with the $\mathrm{O}(2 p)$ states and this can lead to a different hole-magnon vertex and consequently to some redistribution of the $t_{2 g}$-like spectral weight. The other consequence of this intermediate character of $\mathrm{NiO}$ would also be some redistribution of the spectral weight from the actual bound state towards less visible QP's like the
$A$ feature in $\Gamma-M$ direction. Moreover, as the CT gap $\Delta$ is much larger than in the cuprates, one might consider the limit $\Delta \gg U-\Delta$ (with hole-magnon interactions going mainly through $d^{7}$ excitations) and leave the $\sim 1 /(U-\Delta)$ processes unprojected. They lead to an additional " $\sim \widetilde{d}_{\mathbf{k}}^{\dagger} p_{\mathbf{k}-\mathbf{q}} \beta_{\mathbf{q}}$ "' hole-magnon vertex and finite binding energy of a hole at the $\Gamma$ and $M$ points. $^{20,44}$ The resulting Hamiltonian is similar to the recently considered spin-fermion model for $\mathrm{CuO}_{2}$ plane in the mixed-valence regime, ${ }^{44}$ where one could describe not only the oxygen part of the spectrum, but also the copper $3 d^{8}\left({ }^{3} A_{2}\right)$ excited states. Alternatively, one might simplify the effective Hamiltonian neglecting the $t_{2 g}$ orbitals in the model. ${ }^{45}$

In this paper we have actually assumed that the experiment measures a pure $2 p$ spectral weight. The experiment is less perfect and it also picks up appreciable $d$ weight. As we argued, at the $\Gamma$ and $M$ points the nonboundedness of the $2 p$ states is given by symmetry considerations and approved by our calculations. Nevertheless, the experiment shows a large weight in the sector of bound states at this momentum. This can come from the $3 d$ channel $^{36}$ or from the Hubbard corrections to the Green functions, which in the $t-J$ model lead to some redistribution of the spectral weight. ${ }^{13}$ It is expected that also the $3 d$ spectral function would show appreciable weights in the ZR sector. ${ }^{36}$ This should even be true in the strong-coupling limit. As Eskes and Eder have shown, ${ }^{46}$ one has to be careful with wave-function renormalization factors showing up in the strong-coupling expansions. Also in the present case, these are neglected and in parallel with the $t-J$ case they are expected to give rise to disproportionally large spectral weight transfers to the low-lying states. There is still some controversy about the validity of the $t$ - $J$-like models for these systems. ${ }^{47}$ What is needed is a further systematical study of these spectral weight transfer effects in the present spin-fermion model context, as well as an experimental effort aimed at the separation of the pure $2 p$ and $3 d$ spectral weights.

Another effect not included in the present model is the electron-phonon interaction. We have verified that including a small electron-phonon vertex $\left(M \ll J_{K}\right.$, where the vertex was assumed to be independent of the momenta) due to a coupling to an Einstein mode $(\Omega \sim J)$, and using a similar SCBA for both kinds of vertices, ${ }^{48}$ results in even less dispersive QP's than those obtained in Sec. III. This indicates that in order to allow a more quantitative comparison with the experiment, certain imperfections of our spin-fermion model would have to be improved to make it more realistic.

Recently the electronic excitations in $\mathrm{NiO}$ were investigated starting from the Hartree-Fock ${ }^{29}$ or the LDA (Ref. 49) approaches, and taking into account the local three-body correlations. Although the self-energy corrections included in these calculations lead to the correct value of the band gap, they neglect the explicit treatment of the oxygen orbitals and do not include any magnetic scattering, which is the main ingredient of our strong-coupling model. As a result, the low-energy states found by them have a predominantly nickel character, in contrast with the oxygen-bound states found in the present method.

The results obtained for $\mathrm{NiO}$ combined with the earlier ones for the $\mathrm{CuO}_{2}$ plane ${ }^{8}$ encourage us to argue that similar 
physics stands behind the measured photoemission spectra for other strongly correlated transition metal oxides, as, for instance, $\mathrm{CoO}$ (see Ref. 50) or FeO, which have similar crystal and magnetic structure, ${ }^{1}$ and their parameters are believed to represent also the CT regime. ${ }^{19}$

On a more general level, the message of this work is that our calculations suggest an interpretation of the single-hole spectral function of $\mathrm{NiO}$ being different from previous approaches that put more emphasis on local aspects of the problem. Conceptually, it implies that the quantum dynamics at work is of a spatial-temporal character. This is a theme that extends far beyond the present simple context. It underlies such important topics in the field of strongly correlated fermions as the meaning of the infinitely dimensional dynamical mean-field theory in physical dimensions,${ }^{51}$ the relevance of the Anderson impurity viewpoint towards heavyfermion physics, etc. We suspect that the experimental community might find ways to test the precise instances where our picture differs radically from the other interpretations.

\section{ACKNOWLEDGMENTS}

It is a pleasure to thank L. F. Feiner, G. A. Sawatzky, H. Eskes, the late S. Schmitt-Rink, and J. van Elp for valuable discussions. Two of us (J.B. and A.M.O.) acknowledge the support by the Committee of Scientific Research (KBN) of Poland, Project No. 2 P03B 175 14, and J.Z. acknowledges the support by the Foundation of Fundamental Research on Matter (FOM), which is sponsored by the Netherlands Organization for the Advancement of Pure Research (NWO).

\section{APPENDIX: SYMMETRY OF QUASIPARTICLES AT DIFFERENT MOMENTA}

In order to gain more insight into the nature of QP states at different momenta we have examined the self-energy $\Sigma(\mathbf{k}, \omega)$ at two high-symmetry points $(X / 2$ and $Y)$ in the unfolded BZ (Fig. 9). The real part of $\Sigma(\mathbf{k}, \omega)$ changes sign at intermediate energies, being negative (positive) in the low(high-) energy sector. Such a behavior comes from the following relation between the real part of the self-energy and spectral functions:

$$
\operatorname{Re} \Sigma_{\mu \mu}(\mathbf{k}, \omega)=\sum_{\mathbf{q}, \nu} M_{\mu \nu}^{2}(\mathbf{k}, \mathbf{q}) \int_{-\infty}^{\infty} d \omega^{\prime} \frac{A_{\nu}\left(\mathbf{k}-\mathbf{q}, \omega^{\prime}\right)}{\omega-\omega_{\mathbf{q}}-\omega^{\prime}} .
$$

The presence of bound states manifests itself by the minima found for $\omega \simeq-2.0 \mathrm{eV}$ in both $\operatorname{Re} \Sigma(\mathbf{k}, \omega)$ and $\operatorname{Im} \Sigma(\mathbf{k}, \omega)$. In addition to these low-energy states one finds the maxima in $\operatorname{Re} \Sigma(\mathbf{k}, \omega)$ at $\omega \simeq-8.0 \mathrm{eV}$, near the high-energy tiny antibound states along $\Gamma-X / Y$. The strongest oscillations in the self-energy occur at the $X / 2$ point [see Fig. 9(a)] where the bound states are the strongest, whereas smaller changes are found at the $Y$ point, making the spectral features at this point less affected by magnon scattering. The oscillations above the QP energy are less intense than observed in the $t-J$ model, as compared with Figs. 7(c) and 7(d) of Ref. 14.

The relative phases of orbitals at different momenta can be easily obtained by multiplying them with appropriate
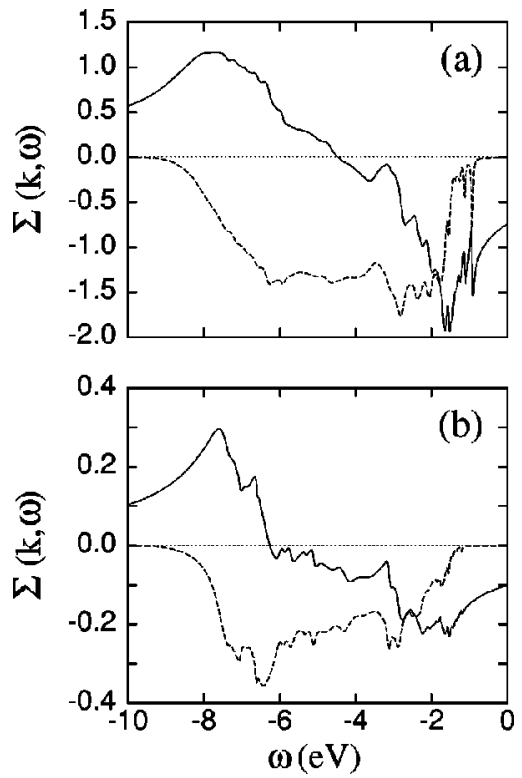

FIG. 9. The hole self-energy for the realistic parameters of $\mathrm{NiO}$ at (a) the $X / 2$ and (b) the $Y$ points in the BZ. Solid and dashed lines correspond to the real and imaginary parts of the self-energy, respectively.

$\exp (i \mathbf{k} \cdot \mathbf{R})$ factors (see Fig. 7). In the presence of direct $p-p$ hoppings one should consider two sets of orthogonal oxygen orbitals (parallel or perpendicular to their nearest neighbors) rather than their linear combinations having the local $d$ symmetry. Moreover, we have to take into account that the umklapp scattering can mix the symmetries belonging to $\mathbf{k}$ and $\mathbf{k}+\mathbf{Q}$ states. To confirm the previous findings concerning the symmetry of bound states at different points in the BZ, we have performed additional calculations for the realistic parameters but with the Kondo element of one of the symmetries switched off in the vertex $M_{\mu \nu}(\mathbf{k}, \mathbf{q})$. We observed substantial alterations in the intensity and positions of main features when $J_{x, e}=0$ or $J_{z, e}=0$ was assumed, whereas for $J_{t}=0$ we found only very slight changes in the spectra.

As far as the $\Gamma-X$ and $\Gamma-Y$ directions are concerned (see the spectra presented in Fig. 6), we have found that the bound state $(A 1)$ has the $x^{2}-y^{2}$ symmetry, while for the remaining spectrum both $x y$ and $3 z^{2}-r^{2}$ symmetries are allowed. In fact, the $C 1 / D 1$ features in the $\Gamma-X / Y$ directions have mainly the $3 z^{2}-r^{2}$ symmetry (very strong change in the intensity of this state was found when $J_{z, e}=0$ ), while weak features $D 1, E 1$ in the $\Gamma-Y$ direction result from the interactions in all three channels. In the $\Gamma-M$ direction [Fig. 5(a)] the low-lying QP state at small momenta and highenergy states close to the $M$ point both have predominantly the $x^{2}-y^{2}$ symmetry. Moreover, with $J_{x, e}=0$ one finds two additional band states at intermediate energies in the $\Gamma-X$ direction that are present in the model without magnons, but are completely wiped out by magnon scattering.

Unlike in the spin-fermion model for the $\mathrm{CuO}_{2}$ plane, ${ }^{8}$ where ferromagnetically correlated $\pi$ states acted like "pairbreakers" on the bound states in the $e_{g}$ channel, here we encounter the FM Kondo coupling being negligible $\left(J_{t}^{2} / J_{x, e}^{2}\right.$ 
$\sim 0.01$ ). This different behavior is naturally explained by different lattice structures. In the $\mathrm{CuO}_{2}$ plane, $e_{g}$ and $t_{2 g}$ bound states are built on different subsets of oxygen orbitals, whereas in $\mathrm{NiO}$ a $\sigma$-like orbital in one direction becomes $\pi$-like in the other. Large overlap between both symmetries combined with a very small $J_{t}$ Kondo element gives that only $e_{g}$ coherent states can be built within our model for $\mathrm{NiO}$.
${ }^{1}$ K. Terakura, T. Oguchi, A. R. Williams, and J. Kübler, Phys. Rev. B 30, 4734 (1984); K. Terakura, A. R. Williams, T. Oguchi, and J. Kübler, Phys. Rev. Lett. 52, 1830 (1984).

${ }^{2}$ A. Fujimori and F. Minami, Phys. Rev. B 30, 957 (1984).

${ }^{3}$ J. Zaanen and G. A. Sawatzky, Prog. Theor. Phys. Suppl. 101, 231 (1990)

${ }^{4}$ Z. X. Shen, C. K. Shih, O. Jepsen, W. E. Spicer, I. Lindau, and J. W. Allen, Phys. Rev. Lett. 64, 2442 (1990).

${ }^{5}$ Z. X. Shen, R. S. List, D. S. Dessau, B. O. Wells, O. Jepsen, A. J. Arko, R. Barttlet, C. K. Shih, F. Parmigiani, J. C. Huang, and P. Lindberg, Phys. Rev. B 44, 3604 (1991).

${ }^{6}$ F. C. Zhang and T. M. Rice, Phys. Rev. B 37, 3759 (1988).

${ }^{7}$ S. Schmitt-Rink, C. M. Varma, and A. E. Ruckenstein, Phys. Rev. Lett. 60, 2793 (1989).

${ }^{8}$ J. Bala, A. M. Oleś, and J. Zaanen, Phys. Rev. B 54, 10161 (1996).

${ }^{9}$ E. Dagotto, Rev. Mod. Phys. 66, 763 (1994).

${ }^{10}$ A. Ramšak and P. Horsch, Phys. Rev. B 48, 10559 (1993); 57, 4308 (1998).

${ }^{11}$ C. L. Kane, P. A. Lee, and N. Read, Phys. Rev. B 39, 6880 (1989)

${ }^{12}$ K. J. von Szczepanski, P. Horsch, W. Stephan, and M. Ziegler, Phys. Rev. B 41, 2017 (1990).

${ }^{13}$ O. P. Sushkov, G. A. Sawatzky, R. Eder, and H. Eskes, Phys. Rev. B 56, 11769 (1997); F. Lema and A. A. Aligia, ibid. 55, 14 092 (1997).

${ }^{14}$ G. Martínez and P. Horsch, Phys. Rev. B 44, 317 (1991).

${ }^{15}$ W. F. Brinkman and T. M. Rice, Phys. Rev. B 2, 1324 (1970).

${ }^{16}$ S. A. Trugman, Phys. Rev. B 37, 1597 (1988).

${ }^{17}$ J. Bala, A. M. Oleś, and J. Zaanen, Phys. Rev. Lett. 72, 2600 (1994).

${ }^{18}$ Our earlier paper (Ref. 17) actually contains an important numerical flaw. We discovered after this paper appeared in press that we had overlooked the umklapp scattering of the hole against the static Néel order parameter. This amounts to a flaw in the zeroth order of the expansion, and leads to large numerical inaccuracies. It will be noted that the present results are quite different, and in fact they compare more favorably with the experiment.

${ }^{19}$ J. Zaanen, G. A. Sawatzky, and J. W. Allen, Phys. Rev. Lett. 55, 418 (1985)

${ }^{20}$ J. Zaanen and A. M. Oleś, Phys. Rev. B 37, 9423 (1988).

${ }^{21}$ C. M. Varma, S. Schmitt-Rink, and E. Abrahams, Solid State Commun. 62, 681 (1987); V. J. Emery, Phys. Rev. Lett. 58, 2794 (1987).

${ }^{22}$ L. F. Feiner, J. H. Jefferson, and R. Raimondi, Phys. Rev. B 53, 8751 (1996).

${ }^{23}$ J. Zaanen, A. M. Oleś, and P. Horsch, Phys. Rev. B 46, 5798 (1992).

${ }^{24}$ J. Zaanen and A. M. Oleś, Phys. Rev. B 48, 7197 (1993).

${ }^{25}$ P. Prelovšek, Phys. Lett. A 126, 287 (1988).

${ }^{26}$ A. Ramšak and P. Prelovšek, Phys. Rev. B 42, 10415 (1990).

${ }^{27}$ J. Bala, A. M. Oleś, and J. Zaanen, Phys. Rev. B 52, 4597 (1995).
${ }^{28}$ J. van Elp, H. Eskes, P. Kuiper, and G. A. Sawatzky, Phys. Rev. B 45, 1612 (1992).

${ }^{29}$ M. Takahashi and J. Igarashi, Phys. Rev. B 54, 13566 (1996).

${ }^{30}$ H. Eskes and J. H. Jefferson, Phys. Rev. B 48, 9788 (1993).

${ }^{31}$ At the $\Gamma$ point the $\mathrm{Ni}(3 d)$ and $\mathrm{O}(2 p)$ states do not hybridize, and the gap between these states is reduced from $\Delta$ to $\Delta-2\left(t_{x x}\right.$ $\left.+t_{y y}\right)$ by the interoxygen hopping.

${ }^{32}$ In the spin-fermion model for $\mathrm{CuO}_{2}$ planes (Ref. 8) we have separated the $\mathbf{k}$ and $\mathbf{k}+\mathbf{Q}$ states by splitting the oxygen bands at the edge of the folded BZ. Here, with larger spin $(S=1)$ and more complicated folding of the bands, such an approximation is no longer reasonable. Instead, we keep the contributions of all four bands in the later self-consistent calculations.

${ }^{33}$ C.-O. Almbladh and L. Hedin, in Handbook on Synchrotron Radiation, edited by E. E. Koch (North-Holland, Amsterdam, 1983), Vol. 1, p. 607; L. Hedin, J. Phys.: Condens. Matter 11, R489 (1999).

${ }^{34}$ J. Bala and A. M. Oleś, Phys. Rev. B 58, 9408 (1998); Physica B 259-261, 449 (1999).

${ }^{35}$ B. O. Wells, Z. X. Shen, A. Matsuura, D. M. King, M. A. Kastner, M. Greven, and R. J. Birgeneau, Phys. Rev. Lett. 74, 964 (1995); J. Pothuizen, R. Eder, N. T. Hien, M. Matoba, A. A. Menovsky, and G. A. Sawatzky, ibid. 78, 717 (1997).

${ }^{36}$ J. Eroles, C. D. Batista, and A. A. Aligia, cond-mat/9812325 (unpublished); Phys. Rev. B 59, 14092 (1999).

${ }^{37}$ V. J. Emery and G. Reiter, Phys. Rev. B 38, 11938 (1988); 41, 7247 (1990).

${ }^{38}$ V. I. Anisimov, M. A. Korotin, J. Zaanen, and O. K. Andersen, Phys. Rev. Lett. 68, 345 (1992); J. Zaanen and P. B. Littlewood, Phys. Rev. B 50, 7222 (1994); Y.-S. Yi, Z.-G. Yu, A. R. Bishop, and J. T. Gammel, ibid. 58, 503 (1998).

${ }^{39}$ X.-X. Bi and P. C. Ecklund, Phys. Rev. Lett. 70, 2625 (1993); C. H. Chen, S.-W. Cheong, and A. S. Cooper, ibid. 71, 2461 (1993); J. M. Tranquada, D. J. Buttrey, V. Sachan, and J. E. Lorenzo, ibid. 73, 1003 (1994).

${ }^{40}$ H. Eskes, M. Meinders, and G. A. Sawatzky, Phys. Rev. Lett. 67, 1035 (1991); H. Eskes and A. M. Oleś, ibid. 73, 1279 (1994); H. Eskes, A. M. Oleś, M. Meinders, and W. Stephan, Phys. Rev. B 50, 17980 (1994).

${ }^{41}$ In the previous paper (Ref. 17) we neglected the umklapp scattering and repaired this flaw by taking an increased value of $t_{\pi}$ by a factor 1.8 (leading to $t_{\pi} \approx t_{x}$ ) and enlarging the oxygen hoppings by a factor 1.2. Including the AF splitting of the oxygen bands, which was previously overlooked, it is no longer necessary to modify the realistic parameters (Ref. 28) so severely to obtain a reasonable agreement with the experimental data.

${ }^{42}$ Similar situation has been found in the frustrated $t-J$ model [J. Bala and A. M. Oleś, Phys. Rev. B 54, 3495 (1996)] where the deformation of magnons do not effect the width of the incoherent part of the spectrum.

${ }^{43}$ J. Bala, A. M. Oleś, and J. Zaanen, Mol. Phys. Rep. 12, 11 (1995). 
${ }^{44}$ J. Bala and A. M. Oleś, Acta Phys. Pol. A 91, 333 (1997).

${ }^{45}$ C. D. Batista and A. A. Aligia, Phys. Rev. B 47, 8929 (1993).

${ }^{46}$ H. Eskes and R. Eder, Phys. Rev. B 54, R14 226 (1996).

${ }^{47}$ C. D. Batista, A. A. Aligia, and J. Eroles, Phys. Rev. Lett. 81, 4027 (1998); E. Dagotto and J. Riera, ibid. 81, 4028 (1998).

${ }^{48}$ A. Ramšak, P. Horsch, and P. Fulde, Phys. Rev. B 46, 14305 (1992).

${ }^{49}$ F. Manghi, C. Calandra, and S. Ossicini, Phys. Rev. Lett. 73, 3129 (1994).
${ }^{50}$ J. van Elp, J. L. Wieland, H. Eskes, P. Kuiper, G. A. Sawatzky, F. de Groot, and T. S. Turner, Phys. Rev. B 44, 6090 (1991); Z.-X. Shen, J. W. Allen, P. Lindberg, D. S. Dessau, B. O. Wells, A. Borg, W. Ellis, J. S. Kang, S.-J. Oh, I. Lindau, and W. E. Spicer, ibid. 42, 1817 (1990).

${ }^{51}$ A. Georges, G. Kotliar, W. Krauth, and M. J. Rozenberg, Rev. Mod. Phys. 68, 13 (1996); M. Fleck, A. I. Lichtenstein, A. M. Oleś, L. Hedin, and V. I. Anisimov, Phys. Rev. Lett. 80, 2393 (1998); M. Fleck, A. I. Lichtenstein, A. M. Oleś, and L. Hedin, Phys. Rev. B 60, 5224 (1999). 